\title{
GROUPOID COHOMOLOGY AND THE DIXMIER-DOUADY CLASS
}

\author{
PAUL S. MUHLY and DANA P. WILLIAMS
}

[Received 14 June 1993-Revised 26 January 1994]

\section{Introduction}

Suppose $A$ is a continuous-trace $\mathrm{C}^{*}$-algebra with spectrum $\hat{A}$. (Throughout, unless otherwise stated, all $\mathrm{C}^{*}$-algebras will be assumed to be separable and all topological spaces and groupoids will be assumed to be locally compact, Hausdorff, and second countable.) In [4], Dixmier and Douady showed how to associate an element $\delta(A)$ in the Cech cohomology group $H^{3}(\hat{A} ; \mathbb{Z})$ with $A$ in such a way that two algebras, $A_{1}$ and $A_{2}$, are stably isomorphic if and only if $\delta\left(A_{1}\right)=\delta\left(A_{2}\right)$. The element $\delta(A)$ has therefore come to be known as the Dixmier-Douady class of $A$. It is well known that every class in $H^{3}(\hat{A} ; \mathbb{Z})$ can arise as a $\delta(A)$ and that $\delta(A)=0$ precisely when $A$ is stably isomorphic to $C_{0}(\hat{A})$. $($ See $[17, \S 3]$ for more details and further references. As is customary, we identify $H^{3}(\hat{A} ; \mathbb{Z})$ with $H^{2}(\hat{A}, \mathscr{S})$, where $\mathscr{S}$ denotes the sheaf of germs of continuous $\mathbb{T}$-valued functions on $\hat{A}$.) Suppose, now, that $A$ is the $\mathrm{C}^{*}$-algebra of a locally

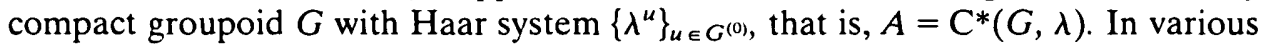
contexts, in recent years, the problems of deciding when $\mathrm{C}^{*}(G, \lambda)$ has continuous trace and identifying its Dixmier-Douady class has arisen (cf., for example, $[10,11,12,14,15,6,7])$. In $[11]$, we showed that if $R$ is a principal groupoid, then $\mathrm{C}^{*}(R, \lambda)$ is a continuous-trace algebra if and only if the usual action of $R$ on its unit space $X$ is proper. (A principal groupoid is essentially an equivalence relation on its unit space. Consequently, we have made the consistent notational convention of denoting them by $R$. Further, when discussing a relation $R$ on a space $X$, we shall simply refer to the unit space of $R$ as $X$.) In this event, $\delta\left(\mathrm{C}^{*}(R, \lambda)\right)=0$, because as we also showed in [11], $\left(\mathrm{C}^{*}(R, \lambda)\right)^{\wedge}$ is homeomorphic to the quotient space of $R$-equivalence classes $X / R$ with the quotient topology, and $C^{*}(R, \lambda)$ is stably isomorphic to $C_{0}(X / R)$. On the other hand, for certain principal groupoids $R$, it is possible to find a groupoid 2-cocycle $\sigma$ such that the twisted groupoid $\mathrm{C}^{*}$-algebra determined by $\sigma$ (and $\left.\lambda\right) \mathrm{C}^{*}(R, \sigma, \lambda)$ in the sense of Renault [18] has continuous trace with non-zero Dixmier-Douady class [14]. Indeed, as Raeburn and Taylor show, there is a very tight relation between Cech cohomology and groupoid cohomology in the particular setting they considered. They showed that given a locally compact space $Y$, and an element $\delta \in H^{2}(Y, \mathscr{S})$, one can build a relation $R$ on a space $X$ such that $X / R$ is homeomorphic to $Y$ and one can construct an explicit $\sigma \in H^{2}(R, \pi)$ from $\delta$ so that the Dixmier-Douady class of $C^{*}(R, \sigma, \lambda)$ is $\delta$ for any choice of $\lambda$. This, then, begs the question of what

This research was partially supported by the Australian Research Council and the National Science Foundation.

1991 Mathematics Subject Classification: 46L05, 46L35.

Proc. London Math. Soc. (3) 71 (1995) 109-134. 
the precise relation is between groupoid cohomology and topological cohomology.

A fairly complete answer was given by Kumjian [6] under the hypothesis that $R$ is an $r$-discrete principal groupoid in the sense of Renault [18]. To state his answer, one must recognize that a 2-cocycle on $R$ gives rise to a certain extension of $R$ by the groupoid $X \times \mathbb{T}$ and it turns out to be important to consider all extensions of $R$ by this groupoid. Such extensions may be characterized as principal $\mathbb{T}$-bundles over $R$ satisfying certain conditions and, consequently, they are called $\mathbb{T}$-groupoids. A $\mathbb{T}$-groupoid comes from a 2-cocycle $\sigma \in H^{2}(R, \mathbb{T})$ precisely when it is trivial as a bundle over $R$ [7]. The collection of isomorphism classes of $\pi$-groupoids over $R$ becomes a group under the usual operations used to define groupoid extensions $[\mathbf{1 8}, \mathbf{7}]$ and we denote this group by $\operatorname{Tw}(R)$ because $\nabla$-groupoids are sometimes also called twists. Given a $\nabla$-groupoid $G$ over $R$ and a Haar system $\left\{\lambda^{u}\right\}_{u \in X}$ on $R$, one constructs a $C^{*}$-algebra $C^{*}(R ; G, \lambda)$ which is a quotient of $\mathrm{C}^{*}(G, \tilde{\lambda})$, where $\tilde{\lambda}$ is a Haar system on $G$ induced by $\lambda$; if $G$ comes from a 2-cocycle $\sigma$, then $\mathrm{C}^{*}(R ; G, \lambda)=\mathrm{C}^{*}(R, \sigma, \lambda)$. In [12], we proved that $\mathrm{C}^{*}(R ; G, \lambda)$ is a continuous-trace $\mathrm{C}^{*}$-algebra precisely when the action of $R$ on $X$ is proper. In this case, then, there is a naturally defined map $\delta: \operatorname{Tw}(R) \rightarrow$

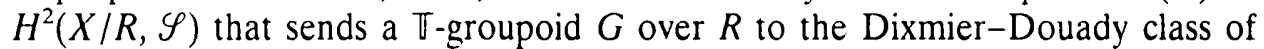
$\mathrm{C}^{*}(R ; G, \lambda)$. Our objective in this paper is to illuminate the properties of this map. Our main result requires the hypothesis that the natural map $p: X \rightarrow X / R$ admits local sections in the sense that given $u \in X / R$, there are a neighbourhood $U$ of $u$ in $X / R$ and a continuous map $\omega: U \rightarrow X$ such that $p \circ \omega(v)=v$, for all $v \in U$. This hypothesis is satisfied in many situations of interest.

The following theorem, which is the main result of this paper, was proved by Kumjian in [6] under the hypothesis that $p: X \rightarrow X / R$ is a local homeomorphism. Our result is more general than his and our proofs are different at certain critical stages. They utilize heavily the notion of the imprimitivity groupoid of a proper groupoid action. (See $\$ 3$ for the development of this notion.)

THEOREM 1.1. Suppose that the relation $R$ on $X$ acts properly and admits a Haar system. Suppose, too, that the quotient map $p: X \rightarrow X / R$ admits local sections. Then there results an exact sequence

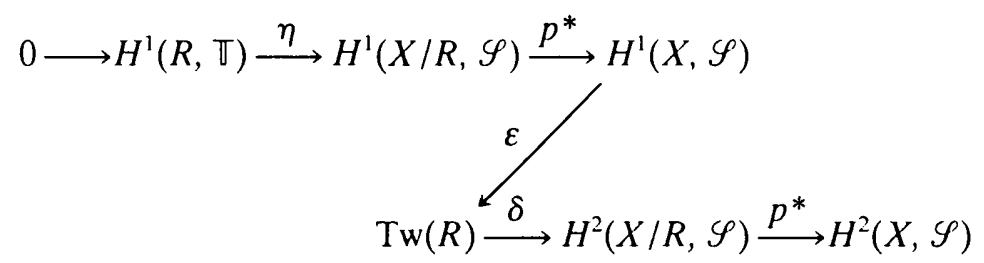

The first group is the first groupoid cohomology group of $R$ with values in $T$ and the second is the first Cech cohomology group with values in the sheaf $\mathscr{Y}$. We think of elements in the latter as defining circle bundles over $X / R$. Cocycles for $R$ with values in $\mathbb{T}$ give rise naturally to circle bundles on $X / R$. The map $\eta$ comes from mapping a cocycle to the corresponding bundle. The two occurrences of $p *$ are simply the natural pull-back maps on Čech cohomology that $p$ induces. The map $\delta$ has already been discussed and its properties will be amplified later. 
Finally, the map $\varepsilon$ arises from viewing elements in $H^{1}(X, \mathscr{S})$ as circle bundles on $X$. Given such a bundle, $\Lambda$, one views it as a proper $X \times \mathbb{T}$ space. The image of $\Lambda$ under $\varepsilon$ is simply the imprimitivity groupoid of this action restricted to $R$. Imprimitivity groupoids play a crucial role in analysing the second occurrence of $p^{*}$, too.

As an immediate corollary of our main theorem and the usual identification of $H^{n+1}(Y ; \mathbb{Z})$ with $H^{n}(Y, \mathscr{S})$, it is interesting to note that if the cohomology of $X$ is trivial, then, at least in dimensions one and two, the groupoid cohomology of $R$ coincides with the ordinary integral cohomology of $X / R$ (in dimensions two and three). More precisely:

Corollary 1.2. Suppose that, in addition to the hypotheses in Theorem 1.1, $H^{1}(X, \mathscr{S})=0=H^{2}(X, \mathscr{S})$. Then $H^{1}(R, \mathbb{T})$ is isomorphic to $H^{1}(X / R, \mathscr{S})$, and $\operatorname{Tw}(R)$ is isomorphic to $H^{2}(X / R, \mathscr{S})$.

The next section is devoted to some topological preliminaries. In $\S 3$, we consider $\mathbb{T}$-groupoids and $\mathbb{T}$-groupoid equivalences in the sense of [9]. It is here that we formally define imprimitivity groupoids, although the notion is implicit in [9] and [20]. In $\S 4$, we attend to the first two terms in the sequence. In $\S 5$, we show that the kernel of $\delta$ coincides with the range of $\varepsilon$. Finally, in $\S 6$, we show that the sequence is exact at $H^{2}(X / R, \mathscr{S})$.

Acknowledgments. The authors were partially supported by the National Science Foundation and by the Australian Research Council. We wish to thank Iain Raeburn and his colleagues at the University of Newcastle for their warm hospitality and friendship during our stay there.

We also would like to thank both the referee and Jean Renault for calling our attention to Renault's paper [19] which has points of contact with our paper. Very roughly speaking, Renault builds Morita equivalence into the group of twists, $\operatorname{Tw}(R)$, and identifies the Dixmier-Douady invariant of the $\mathrm{C}^{*}$-algebra associated with an element in his $\operatorname{Tw}(R)$ with its image, under a naturally defined homomorphism, in $H^{2}(X / R, \mathscr{S})$.

\section{Topological preliminaries}

We will always assume that our groupoids are locally compact, Hausdorff, and have open range and source maps, $r$ and $s$. The hypothesis on $r$ and $s$ is automatically satisfied if the groupoid in question admits a Haar system [18, Proposition I.2.4], and these are the only sorts of groupoids we will be interested in here.

If $G$ is a groupoid, and if $A \subseteq G^{(0)}$, then we will write $G_{A}=s^{-1}(A)$ and $G^{A}=r^{-1}(A)$. Also, if $C, D \subseteq G$, then we write

$$
C * D=\{(\gamma, \beta) \in C \times D: s(\gamma)=r(\beta)\} .
$$

Fibred products of this sort appear throughout this note; most particularly when considering groupoid actions.

For the definition of these, let $G$ be a groupoid and let $X$ be a topological space together with a continuous open map $\sigma$ from $X$ onto $G^{(0)}$. Form $X * G=$ $\{(x, \gamma): \sigma(x)=r(\gamma)\}$. To say that $G$ acts (continuously) on the right of $X$ means 
that there is a continuous map from $X * G$ to $X$, with the image of $(x, \gamma)$ denoted $x \cdot \gamma$, such that the following hold:

(1) $\sigma(x \cdot \gamma)=s(\gamma)$

(2) $x \cdot(\alpha \beta)=(x \cdot \alpha) \cdot \beta$, for all $(\alpha, \beta) \in G^{(2)}$, and

(3) $x \cdot \sigma(x)=x$ for all $x \in X$.

We think of $\sigma$ as being a 'generalized source' map for the action. Likewise a (continuous) left action of $G$ on $X$ is determined by a continuous open surjection $\rho: X \rightarrow G^{(0)}$, and a continuous map from $G * X$ to $X$ satisfying the appropriate analogues of (1), (2) and (3). In a similar fashion, we think of $\rho$ as a 'generalized range' map. When we speak of left or right actions in the sequel, the maps $\sigma$ and $\rho$ will be implicitly understood and will be referred to without additional comment. Most of our definitions will be made in terms of right actions, but the left-handed versions can be formulated with no difficulty.

Of special importance will be the actions of $G$ on its unit space $G^{(0)}$. Here $\rho$ and $\sigma$ are the identity maps and $r(\gamma) \cdot \gamma=s(\gamma)$ and $\gamma \cdot s(\gamma)=r(\gamma)$.

As with group actions, if $G$ acts on $X$ (on the right, say), and if $x \in X$, the orbit of $x$ is simply $\left\{x \cdot \gamma: \gamma \in G^{\sigma(x)}\right\}$. The orbits partition $X$ and we write $X / G$ for the quotient space with the quotient topology.

Lemma 2.1. If $X$ is a right $G$-space, then the natural map $p: X \rightarrow X / G$ is open.

Proof. Suppose that $V$ is open in $X$. To show that $p(V)$ is open it suffices to show that $V \cdot G$ is open in $X$. Thus it suffices to show that any net $x_{n} \rightarrow x \cdot \gamma$ with $x \in V$ and $\gamma \in G$ has a subnet which is eventually in $V \cdot G$. But $\sigma\left(x_{n}\right) \rightarrow \sigma(x \cdot \gamma)=s(\gamma)$. Since $s$ is open, we may pass to a subnet and assume that there are $\gamma_{n} \rightarrow \gamma$ in $G$ with $s\left(\gamma_{n}\right)=\sigma\left(x_{n}\right)$. Then $x_{n} \gamma_{n}^{-1}$ is eventually in $V\left(x_{n} \gamma_{n}^{-1} \rightarrow x \in V\right)$, and $x_{n}=x_{n} \cdot \gamma_{n}^{-1} \gamma_{n}$ is eventually in $V \cdot G$.

Consider a (right) action of $G$ on $X$ and let $\Psi: X * G \rightarrow X \times X$ be defined by the formula $\Psi(x, \gamma)=(x, x \cdot \gamma)$. Then we call the action free if $\Psi$ is one-to-one. Alternatively, the action is free precisely when the equation $x \cdot \gamma=x$ implies that $\gamma=\sigma(x)$. The action is called proper provided that $\Psi$ is a proper map. The following lemma will be used at a number of points in the sequel. It is proved in [11] under the assumption that $G$ is a principal groupoid acting on $G^{(0)}$. However, the proof presented there works in the generality in which we are interested and so will not be repeated.

LEMMA 2.2. The following conditions on a free (right) action are equivalent:

(1) the action is proper; that is, $\Psi$ is a proper map;

(2) $\Psi$ is a closed map;

(3) $\Psi$ is a homeomorphism onto a closed subset of $X \times X$;

(4) given a compact subset $K \subseteq X$, the set $G(K)=\{\gamma \in G: K \cdot \gamma \cap K \neq \varnothing\}$ is compact;

(5) for all compact sets $K \subseteq X, G(K)$ is relatively compact in $G$.

A key consequence of Lemma 2.2 is

COROLlaRY 2.3. If $G$ acts freely and properly on a locally compact Hausdorff space $X$, then the quotient space, $X / G$, is locally compact and Hausdorff. 
Remark 2.4. Taken together, Lemmas 2.1 and 2.2 and Corollary 2.3 will be used repeatedly throughout this paper. A special application of them deserves to be singled out. Normally one thinks of a principal T-bundle $S$ over a space $X$ as a locally trivial fibre bundle with fibre $\mathbb{T}$ determined by transition functions taking values in $\mathbb{T}$. These bundles are classified up to isomorphism by $H^{1}(X, \mathscr{S})$, where $\mathscr{S}$ is the sheaf of continuous $\mathbb{T}$-valued functions. However, thanks to [5] and the results just presented, to show that a locally compact space $S$ is a principal $\pi$-bundle over a locally compact space $X$ determined by a surjection $\tau: S \rightarrow X$, it suffices to check that $\tau$ is continuous and open, and that $S$ carries a free $\pi$-action such that $\tau(\xi)=\tau(\eta)$ if and only if $t \cdot \xi=\eta$ for some (necessarily unique) $t$ in $T$. (Note that actions of compact groups are automatically proper.) The assumptions on $\tau$ allow us to identify $X$ with $S / \pi$ and $\tau$ with the quotient map.

Recall that a transversal is a subset $F \subseteq G^{(0)}$ which meets each equivalence class in $G^{(0)} / G$ exactly once. We shall be exclusively interested in closed transversals obtained from continuous cross-sections $w$ for $p: G^{(0)} \rightarrow G^{(0)} / G$; if $w$ is a continuous cross-section, then $F=w\left(G^{(0)} / G\right)$ is a closed transversal.

Notice that if $F$ is a closed transversal, then $\left.s\right|_{G_{r}}$ is an open map from $G_{F}$ to $F$. On the other hand, $\left.r\right|_{G_{F}}$, which is a surjection of $G_{F}$ onto $G^{(0)}$ since $F$ is a transversal, need not be open in general [9, Example 2.2]. Our first task is to find general conditions which will ensure that $\rho=\left.r\right|_{G_{F}}$ is open for each transversal $F$ as above. Our next result is a generalization of [9, Theorem 2.2A].

THEOREM 2.5. Suppose that $G$ is a locally compact groupoid with open range and source maps $r$ and $s$. Also assume that there is a continuous cross-section $w$ for $p: G^{(0)} \rightarrow G^{(0)} / G$ and let $F$ be the associated transversal. Then the following statements are equivalent:

(1) the map $\Phi: G_{F} * G^{F} \rightarrow G$, defined by $\Phi(\gamma, \beta)=\gamma \beta$, is an open surjection;

(2) the map $\rho=\left.r\right|_{G_{r}}: G_{F} \rightarrow G^{(0)}$ is an open surjection;

(3) the map $\pi: G \rightarrow G^{(0)} \times G^{(0)}$, defined by $\pi(\gamma)=(r(\gamma)$, s( $\gamma)$ ), is an open map onto its range.

(Note that in (3) we are not requiring $\pi(G)$ to be open in $G^{(0)} \times G^{(0)}$ !)

Proof. We will show that $(3) \Leftrightarrow(2) \Leftrightarrow(1)$. We start with (3) $\Rightarrow(2)$. Let $V$ be open in $G$. It will suffice to show that

$$
\rho\left(V \cap G_{F}\right)=r\left(V \cap G_{F}\right)
$$

is open in $G^{(0)}$. Suppose that $u=r(\gamma)$ with $\gamma \in V \cap G_{F}$. It will be sufficient to show that if $u_{n} \rightarrow u$ is any net in $G^{(0)}$, then it has a subnet which is eventually in $r\left(V \cap G_{F}\right)$. But $p\left(u_{n}\right) \rightarrow p(u)$ in $G^{(0)} / G$, so, using our cross-section $w$, we see that there are $v_{n}, v \in F$ such that $v_{n} \sim u_{n}, v \sim u$, and $v_{n} \rightarrow v$. Thus, $\left(u_{n}, v_{n}\right) \rightarrow(u, v)$ in the range of $\pi$. Since we are assuming that $\pi$ is open, we can pass to a subnet, relabel, and assume that there are $\gamma_{n} \in G$ with $\gamma_{n} \rightarrow \gamma$ such that $r\left(\gamma_{n}\right)=u_{n}$ and $s\left(\gamma_{n}\right)=v_{n}$ Notice that each $\gamma_{n} \in G_{F}$ by construction and $\left\{\gamma_{n}\right\}$ is eventually in $V$ as $V$ is open and $\gamma \in V$. Therefore $u_{n}$ is eventually in $r\left(V \cap G_{F}\right)$.

Next we consider (2) $\Rightarrow(1)$. It is useful to realize $\Phi$ as the composition of the homeomorphism $(\alpha, \beta) \mapsto(\alpha, \alpha \beta)$ of $G_{F} * G^{F}$ onto

$$
G_{F} \times{ }_{r} G=\left\{(\gamma, \beta) \in G_{F} \times G: r(\gamma)=r(\beta)\right\}
$$

with the projection $\mathrm{pr}_{2}$ on the second factor. Therefore, it will suffice to show 
that the projection is open. But if $W$ and $V$ are open in $G$, then we claim that

$$
\operatorname{pr}_{2}\left(W \times V \cap G_{F} \times{ }_{r} G\right)=r^{-1}\left(\rho\left(W \cap G_{F}\right)\right) \cap V .
$$

To see this, suppose that $\beta$ belongs to the left-hand side. Then there is an $\alpha \in W \cap G_{F}$ with $r(\alpha)=r(\beta)$. Thus $\beta \in r^{-1}\left(\rho\left(W \cap G_{F}\right)\right) \cap V$. On the other hand, if $\beta$ belongs to the right-hand side, then $\beta \in V$ and $r(\beta) \in \rho\left(W \cap G_{F}\right)$. Let $\alpha \in W \cap G_{F}$ satisfy $r(\alpha)=r(\beta)$. Then $(\alpha, \beta) \in W \times V \cap G_{F} \times{ }_{r} G$. This establishes (2.1); consequently, $\operatorname{pr}_{2}$ is open when $\rho$ is.

Next we show that $(1) \Rightarrow(2)$. Consider the commutative diagram

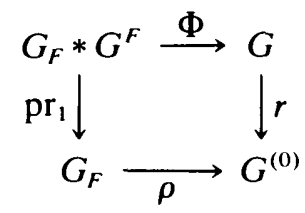

Since $\mathrm{pr}_{1}$ is continuous and surjective, and since $r$ is always assumed to be open, $\rho$ is open whenever $\Phi$ is.

Now assume (2). Notice that if $\gamma, \beta \in G_{F}$ satisfy $\rho(\gamma)=\rho(\beta)$, then $s(\gamma) \sim$ $r(\gamma)=r(\beta) \sim s(\beta)$. Since $F$ is a transversal, $s(\gamma)=s(\beta)$. Therefore $\beta^{-1} \gamma \in G_{F}^{F}$. Since the natural map from $G_{F}$ onto $G_{F} / G_{F}^{F}$ is open by Lemma 2.1 , it follows that, if $\rho$ is open, then $\rho$ induces a homeomorphism of $G_{F} / G_{F}^{F}$ onto $G^{(0)}$. Since inversion is a homeomorphism on $G$, we also have $\left.s\right|_{G^{F}}$ inducing a homeomorphism of $G_{F}^{F} \backslash G^{F}$ onto $G^{(0)}$. We shall write $\psi$ for the homeomorphism of $G_{F} / G_{F}^{F} \times G_{F}^{F} \backslash G^{F}$ onto $G^{(0)} \times G^{(0)}$. If $\lambda$ is the natural map from $G_{F} * G^{F}$ onto $G_{F} / G_{F}^{F} \times G_{F}^{F} \backslash G^{F}$, then we have a commutative diagram

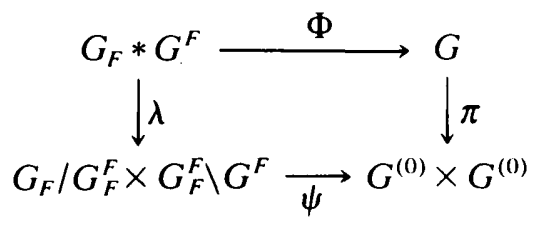

Since $\Phi$ is surjective, $\pi(V)=\psi\left(\lambda\left(\Phi^{-1}(V)\right)\right)$. Thus as $\psi$ is a homeomorphism, it will suffice to show that $\lambda$ is an open map onto its range. In fact, if $\lambda\left(\gamma_{n}, \beta_{n}\right) \rightarrow \lambda(\gamma, \beta)$, then passing to subnets and relabelling, we may assume that there are $\gamma_{n}^{\prime}, \beta_{n}^{\prime} \in G_{F}^{F}$ such that $\gamma_{n} \gamma_{n}^{\prime} \rightarrow \gamma$ and $\beta_{n}^{\prime} \beta_{\eta} \rightarrow \beta$. However, since $F$ is a transversal, $\alpha \in G_{F}^{F}$ implies that $r(\alpha)=s(\alpha)$. Thus $\left(\gamma_{n} \gamma_{n}^{\prime}, \beta_{n}^{\prime} \beta_{n}\right) \rightarrow(\gamma, \beta)$ in $G_{F} * G^{F}$. Thus $\lambda$ is open onto its range and (2) $\Rightarrow(3)$.

COROLlary 2.6. Suppose that $G$ is a second countable locally compact groupoid with abelian stability groups and for which there is a continuous cross-section for the orbit map p: $G^{(0)} \rightarrow G^{(0)} / G$. If both $G$ and the stability groupoid have Haar systems $\lambda$ and $\sigma$, and if any of the equivalent conditions in Theorem 2.5 are satisfied, then $\mathrm{C}^{*}(G, \lambda)$ has continuous trace with trivial Dixmier-Douady class.

Proof. We have $G$ equivalent to $G_{F}^{F}$ by assumption [9, Example 2.7]. Thus $\mathrm{C}^{*}(G, \lambda)$ is (strongly) Morita equivalent to the abelian $\mathrm{C}^{*}$-algebra $\mathrm{C}^{*}\left(G_{F}^{F}, \sigma\right)$ by [9, Theorem 2.8]. Now the result follows from, for example, [17, Proposition 3.1 and Theorem 3.5]. 


\section{T-groupoids}

In this section we will collect some facts about $\mathbb{T}$-groupoids which we will use in the sequel. One of the main results is Theorem 3.2, below. While it is a special case of Corollaire 5.4 of [20], we provide a proof for two reasons. First, it is but a simple variant of Theorem 2.8 of [9]. Corollaire 5.4 of [20] appears at the end of a daunting level of technical development, while Theorem 3.2 lies near the surface. We believe it is useful to see this. Second, and more important, the proof of Corollaire 5.4 presented in [20] cites results of [9] concerning what we call below the imprimitivity groupoid of a principal $G$-space. Only the algebraic properties of the imprimitivity groupoid are developed in [9], but the topological properties are used in [20] and they are used in this paper. We develop them in detail in Theorem 3.5. We begin at a slightly greater level of generality than we need, but the excess will require no additional effort.

Let $G$ be a second countable locally compact groupoid with unit space $X$. Suppose that $\mathbb{T}$ acts on $G$ (on the left), making $G$ a principal $\mathbb{T}$-bundle over $G / \mathbb{T}$. Suppose further that the quotient space $G / \pi$, with the quotient topology, has the structure of a locally compact groupoid such that the bundle map $j: G \rightarrow G / \mathbb{T}$ is a groupoid homomorphism. Finally, suppose that the $\mathbb{T}$-action is related to the groupoid structure on $G$ through the equations $r(t \cdot \gamma)=r(\gamma), s(t \cdot \gamma)=s(\gamma)$, and $(t \cdot \gamma)\left(t^{\prime} \cdot \gamma^{\prime}\right)=\left(t t^{\prime}\right) \cdot\left(\gamma \gamma^{\prime}\right)$ for all $t, t^{\prime} \in \mathbb{T}$ and $\left(\gamma, \gamma^{\prime}\right) \in G * G$. Under these circumstances we call $G$ a $\mathbb{T}$-groupoid over $G / \mathbb{T}$. (See $[\mathbf{7}, 12]$.) The assumptions on the $\mathbb{T}$-action imply that the unit space of $G / \mathbb{T}$ may be identified with $X=G^{(0)}$. So for each $u \in X$ and each $\gamma \in j^{-1}(u)$, there is a unique $t \in \mathbb{T}$ such that $\gamma=t \cdot u$. The map $i: X \times \mathbb{T} . \rightarrow G$ defined by $i(u, t)=t \cdot u$, then, is a homeomorphic homomorphic embedding of the trivial group bundle $X \times \mathbb{T}$ into $G$; in fact the range is contained in $S=\{\gamma: r(\gamma)=s(\gamma)\}$, and we have an exact sequence

$$
X \longrightarrow X \times \mathbb{T} \stackrel{i}{\longrightarrow} G \stackrel{j}{\longrightarrow} G / \mathbb{T} .
$$

Conversely, an exact sequence

$$
X \longrightarrow X \times \mathbb{T} \stackrel{i_{1}}{\longrightarrow} G \stackrel{j_{1}}{\longrightarrow} G_{1}
$$

where $i_{1}$ is a homeomorphism onto its range, which is assumed to be contained in $S$, and $j_{1}$ is a continuous open surjection, enables one to view $G$ as a $\mathbb{T}$-groupoid over $G_{1}$ : simply define $t \cdot \gamma$ to be $i_{1}(r(\gamma), t) \gamma$ for each $\gamma \in G$ and $t \in \mathbb{T}$. Thus, we may think of $\mathbb{T}$-groupoids either in terms of the action of $\mathbb{T}$ on $G$, or in terms of extensions of $X \times \mathbb{J}$ by $G_{1}$. Both perspectives will prove useful. Here we will focus on the case when the range of $i$ is all of $S$, so that $G / \pi$ is a principal groupoid, which we view as the relation $R \subseteq X \times X$ consisting of $\{(r(\gamma), s(\gamma)): \gamma \in G\}$.

Given a $\mathbb{T}$-groupoid $G$ with Haar system $\lambda$ on $G / \mathbb{T}$, one obtains a Haar system $\tilde{\lambda}$ on $G$ defined by the formula

$$
\int f(\gamma) d \tilde{\lambda}^{u}(\gamma)=\iint f(t \cdot \gamma) d t d \lambda^{u}(\dot{\gamma})
$$

where $d t$ is (normalized) Lebesgue measure on $\mathbb{T}$ and $\dot{\gamma}$ denotes $j(\gamma)$. Let 
$C_{c}(G / \mathbb{T} ; G, \lambda)=\left\{f \in C_{c}(G): f(t \cdot \gamma)=t f(\gamma)\right\}$. Then $C_{c}(G / \mathbb{T} ; G, \lambda)$ becomes a *-algebra through the formulas

$$
f * g(\gamma)=\int f(\alpha) g\left(\alpha^{-1} \gamma\right) d \lambda^{r(\gamma)}(\dot{\alpha})
$$

and

$$
f^{*}(\gamma)=\overline{f\left(\gamma^{-1}\right)}
$$

As Renault shows in [18], this algebra may be completed to obtain a $C^{*}$-algebra, which we denote $\mathrm{C}^{*}(G / \mathbb{\pi} ; G, \lambda)$ and call the $\mathbb{T}$-groupoid $\mathrm{C}^{*}$-algebra determined by $G$. It is a very special case of what Renault called a restrained crossed product. Define $\rho_{G}: C_{c}(G, \tilde{\lambda}) \rightarrow C_{c}(G / \mathbb{T} ; G, \lambda)$ by $\rho_{G}(f)(\gamma)=\int f(t \cdot \gamma) \bar{t} d t$. Then since representations of $C_{c}(G, \tilde{\lambda})$ and $C_{c}(G / \pi ; G, \lambda)$ can be disintegrated [20], it is easy to see that $\rho_{G}$ extends to a $C^{*}$-homomorphism from $\mathrm{C}^{*}(G, \tilde{\lambda})$ onto $C^{*}(G / \mathbb{T} ; G, \lambda)$. In $[\mathbf{1 2}$, Theorems 4.2 and 4.3$]$, we showed that if $S=i(X \times \mathbb{T})$, so that $G / \pi$ can be identified with $R$, then $C^{*}(R ; G, \lambda)$ has continuous trace if and only if $R$ is a proper principal groupoid.

In this paper we must deal extensively with questions of (strong) Morita equivalence between $\mathbb{T}$-groupoid $\mathrm{C}^{*}$-algebras and we will have need for the following variation on the main theme of [9]. Suppose that $G$ and $H$ are $\mathbb{T}$-groupoids over $G / \mathbb{T}$ and $H / \mathbb{T}$, respectively, and suppose that $Z$ is a left $G$ - and right $H$-space that implements an equivalence between $G$ and $H$ as groupoids in the sense of [9]. (We will follow the notation in [9]). The actions of $G$ and $H$ induce $t w o$ actions of $\mathbb{T}$ on $Z$. The one coming from $G$ is given by the formula $t \cdot{ }_{G} z=i_{G}(\rho(z), t) \cdot z$, where $\rho: Z \rightarrow G^{(0)}$ is the map used to define the $G$-action on $Z$, and $i_{G}$ is the embedding of $G^{(0)} \times \mathbb{T}$ into $G$ described above. The $\mathbb{T}$-action coming from $H$ is given by a similar formula: $z \cdot{ }_{H} t=x \cdot i_{H}(\sigma(z), t)$.

Definition 3.1. If $G$ and $H$ are $\mathbb{T}$-groupoids and if $Z$ is a $(G, H)$-equivalence in the sense of [9], then we call $Z$ a $(G, H)$ - T-equivalence in the case where the $T$-actions on $Z$ induced by the $G$ and $H$ actions coincide; that is, when $t \cdot G z=z \cdot{ }_{H} t$ for all $z \in Z$ and $t \in \mathbb{T}$.

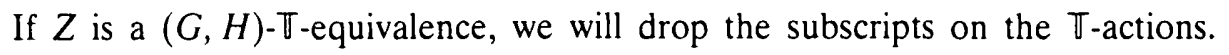
Our goal is to prove

THEOREM 3.2. Suppose that $G$ and $H$ are second countable, locally compact $\mathbb{T}$-groupoids and that $Z$ is a $(G, H)-\mathbb{T}$-equivalence. Let

$$
C_{c}^{\mathbb{T}}(Z)=\left\{\phi \in C_{c}(Z): \phi(t \cdot z)=t \phi(z) \text { for all } z \in Z \text { and } t \in \mathbb{T}\right\} .
$$

Given any Haar systems $\lambda$ on $G / \mathbb{T}$ and $\beta$ on $H / \pi, C_{c}^{\mathbb{\pi}}(Z)$ can be completed naturally to become a $\mathrm{C}^{*}(G / \mathbb{\pi} ; G, \lambda)-\mathrm{C}^{*}(H / \mathbb{\pi} ; H, \beta)$-equivalence bimodule. In particular, $\mathrm{C}^{*}(G / \mathbb{T} ; G, \lambda)$ and $\mathrm{C}^{*}(H / \mathbb{T} ; H, \beta)$ are (strongly) Morita equivalent.

Proof. All that is necessary, really, is to make some minor addenda to the proof of Theorem 2.8 in [9]. To that end, let $A$ be the pre-C*-algebra $C_{c}(G, \tilde{\lambda})$ where $\tilde{\lambda}$ is the Haar system of $G$ determined by $\lambda$ obtained by crossing $\lambda$ with Lebesgue measure on $\mathbb{T}$ as described above. Likewise, let $B=C_{c}(H, \tilde{\beta})$. Let 
$A_{1}=C_{c}(G / \pi ; G, \lambda)$ and $B_{1}=C_{c}(H / \pi ; H, \beta)$ and let $\rho_{G}: A \rightarrow A_{1}$ and $\rho_{H}: B \rightarrow$ $B_{1}$ be the *-homomorphisms determined by 'integrating over $\mathbb{T}$ ' described above. These maps are projections. Likewise, let $\rho_{Z}: C_{c}(Z) \rightarrow C_{c}^{\top}(Z)$ be defined by the formula $\rho_{Z}(f)(z)=\int_{\mathbb{T}} \phi(t \cdot z) \bar{t} d t$. Then $\rho_{Z}$ is a projection from $C_{c}(Z)$ onto $C_{c}^{\top}(Z)$ that is continuous with respect to the inductive limit topology. Recall from p. 11 of [9] that $C_{c}(Z)$ is given the structure of an $A-B$-bimodule via the equations

$$
f \cdot \phi(z)=\int_{G} f(\gamma) \phi\left(\gamma^{-1} \cdot z\right) d \tilde{\lambda}^{\rho(z)}(\gamma)
$$

and

$$
\phi \cdot g(z)=\int_{H} \phi(z \cdot \eta) g\left(\eta^{-1}\right) d \tilde{\beta}^{\sigma(z)}(\eta) .
$$

Observe that if $f \in A_{1}$ and $\phi \in C_{c}^{\top}(Z)$, then $f \cdot \phi$ lies in $C_{c}^{\pi}(Z)$ and (3.1) yields

$$
f \cdot \phi(z)=\int_{G} f(\gamma) \phi\left(\gamma^{-1} \cdot z\right) d \lambda^{\rho(z)}(\dot{\gamma})
$$

The point is that the action passes to the quotient, $\bar{\lambda}$ is replaced by $\lambda$, and $\gamma$ by $\dot{\gamma}$. Likewise, for $g \in B_{1}$ and $\phi \in C_{c}^{\top}(Z), \phi \cdot g$ lies in $C_{c}^{\top}(Z)$ and (3.2) yields

$$
\phi \cdot g(z)=\int_{H} \phi(z \cdot \eta) g\left(\eta^{-1}\right) d \beta^{\sigma(z)}(\dot{\eta}) .
$$

Furthermore, the equations

$$
\rho_{Z}(f \cdot \phi)=f \cdot \rho_{Z}(\phi)
$$

and

$$
\rho_{Z}(\phi \cdot g)=\rho_{Z}(\phi) \cdot g
$$

are satisfied for all $f \in A_{1}, g \in B_{1}$, and $\phi \in C_{c}(Z)$. Recall, too, that there are $A$ and $B$-valued inner products defined on $C_{c}(Z)$ given by the following formulas (the formula on p. 12 of [9] for ${ }_{A}\langle\phi, \psi\rangle$ contains a misprint; the correct formula is (3.8)):

$$
\langle\phi, \psi\rangle_{B}(\eta)=\int_{G} \overline{\phi\left(\gamma^{-1} \cdot z\right)} \psi\left(\gamma^{-1} \cdot z \cdot \eta\right) d \bar{\lambda}^{\rho(z)}(\gamma)
$$

and

$$
{ }_{A}\langle\phi, \psi\rangle(\gamma)=\int_{H} \phi\left(\gamma \cdot z \cdot \eta \overline{\psi(z \cdot \eta)} d \tilde{\beta}^{\sigma(z)}(\eta),\right.
$$

where $\phi, \psi \in C_{c}(Z)$ and the $z$ in (3.7) is chosen so that $\sigma(z)=r(\eta)$, while the $z$ in (3.8) is chosen so that $\rho(z)=s(\gamma)$. It is shown on p. 11 of [9] that the assumption that $Z$ is a $(G, H)$-equivalence guarantees that these equations are independent of the choice of $Z$ and define continuous functions with compact support. The equations (3.7) and (3.8) and our assumption that $Z$ is a $(G, H)$-T-equivalence make it clear that if $\phi$ and $\psi$ are in $C_{c}^{\top}(Z)$, then ${ }_{A}\langle\phi, \psi\rangle$ and $\langle\phi, \psi\rangle_{B}$ are actually in $A_{1}$ and $B_{1}$, respectively. Moreover, since the integrals in each of (3.7) and (3.8) are left unchanged when the variable over which the integration is performed is 
multiplied by $t \in \mathbb{T}$, it is clear that we may rewrite (3.7) and (3.8) and define ${ }_{A_{1}}\langle\phi, \psi\rangle$ and $\langle\phi, \psi\rangle_{B_{1}}$, for $\phi, \psi \in C_{c}^{\mathbb{T}}(Z)$, via the equations

$$
\langle\phi, \psi\rangle_{B_{1}}(\eta)=\int_{G} \overline{\phi\left(\gamma^{-1} \cdot z\right)} \psi\left(\gamma^{-1} \cdot z \cdot \eta\right) d \lambda^{\rho(z)}(\dot{\gamma})
$$

and

$$
{ }_{A_{1}}\langle\phi, \psi\rangle(\gamma)=\int_{H} \phi\left(\gamma \cdot z \eta \overline{\psi(z \cdot \eta)} d \beta^{\sigma(z)}(\dot{\eta}) .\right.
$$

The following equations, valid for $\phi, \psi \in C_{c}(Z)$, are now immediate:

$$
{ }_{A_{1}}\left\langle\rho_{Z}(\phi), \rho_{Z}(\psi)\right\rangle=\rho_{G}\left({ }_{A}\langle\phi, \psi\rangle\right)
$$

and

$$
\left\langle\rho_{Z}(\phi), \rho_{Z}(\psi)\right\rangle_{B_{1}}=\rho_{H}\left(\langle\phi, \psi\rangle_{B}\right)
$$

The next set of equations may now be verified directly or may be proved as simple consequences of the corresponding equations on p. 12 of [9] using equations (3.5), (3.6) (3.11), and (3.12):

$$
\begin{gathered}
f \cdot{ }_{A_{1}}\langle\phi, \psi\rangle={ }_{A_{1}}\langle f \cdot \phi, \psi\rangle, \\
\langle\phi, \psi\rangle_{B_{1}}=\langle\phi, \psi \cdot g\rangle_{B_{1}}, \quad \text { where } f \in A_{1}, \phi, \psi \in C_{c}^{\pi}(Z), \\
\left(f * f_{1}\right) \cdot \phi=f \cdot\left(f_{1} \cdot \phi\right), \quad \text { where } f, f_{1} \in A_{1}, \phi \in C_{c}^{\pi}(Z), \\
g \cdot\left(g * g_{1}\right)=(\phi \cdot g) \cdot g_{1}, \quad \text { where } g, g_{1} \in B_{1}, \phi \in C_{c}^{\pi}(Z), \\
{ }_{A_{1}}\langle\phi, \psi\rangle^{*}={ }_{A_{1}}\langle\psi, \phi\rangle, \quad\langle\phi, \psi\rangle_{B_{1}}^{*}=\langle\psi, \phi\rangle_{B_{1}}, \quad \text { and } \\
{ }_{A_{1}}\langle\phi, \psi\rangle \cdot \xi=\phi \cdot\langle\psi, \xi\rangle_{B_{1}}, \quad \text { for } \phi, \psi, \xi \in C_{c}^{\pi}(Z) .
\end{gathered}
$$

To illustrate the latter approach, observe that

$$
\begin{aligned}
f \cdot{ }_{A_{1}}\langle\phi, \psi\rangle & =\rho_{G}(f) \rho_{C}\left({ }_{A}\langle\phi, \psi\rangle\right)=\rho_{G}\left(f \cdot{ }_{A}\langle\phi, \psi\rangle\right) \\
& =\rho_{G}\left({ }_{A}\langle f \cdot \phi, \psi\rangle\right)={ }_{A_{1}}\left\langle\rho_{Z}(f \cdot \phi), \rho_{Z}(\psi)\right\rangle \\
& ={ }_{A_{1}}\langle f \cdot \phi, \psi\rangle .
\end{aligned}
$$

The positivity of the two inner products follows from (3.11) and (3.12) and the facts that $\rho_{G}$ and $\rho_{H}$ extend to be $C^{*}$-homomorphisms. Likewise, the density of the inner products follows from the corresponding facts about the $A$ - and $B$-valued inner products on $C_{c}(Z)$ and the continuity of the maps $\rho_{G}$ and $\rho_{H}$ in the inductive limit topology. Indeed, for example, as shown in [9], given $g \in B_{1}$ there is a net $\left\{g_{\alpha}\right\}$ in $C_{c}(H, \widetilde{\beta})$ converging to $g$ in the inductive limit topology such that each $g_{\alpha}$ has the form $\sum_{n}\left\langle\phi_{n}^{\alpha}, \psi_{n}^{\alpha}\right\rangle_{B}$. Since $\left\{\rho_{H}\left(g_{\alpha}\right)\right\}$ also converges to $g$, the desired conclusion follows from (3.12). Finally, the boundedness of the inner products is seen to be a consequence of (3.11) and (3.12). For example, for $f \in A_{1}$ and $\phi, \psi \in C_{c}^{\mathbb{\pi}}(Z)$, we have

$$
\begin{aligned}
\langle f \cdot \phi, f \cdot \phi\rangle_{B_{1}} & =\rho_{H}\left(\langle f \cdot \phi, f \cdot \phi\rangle_{B}\right) \leqslant\|f\|_{A}^{2} \rho_{H}\left(\langle\phi, \phi\rangle_{B}\right) \\
& =\|f\|_{A_{1}}^{2}\left\langle\rho_{Z}(\phi), \rho_{Z}(\phi)\right\rangle_{B_{1}}=\|f\|_{A_{1}}^{2}\langle\phi, \phi\rangle_{B_{1}} .
\end{aligned}
$$

We have verified all the conditions necessary to show that $C_{c}^{\pi}(Z)$ may be 
completed to form a $\mathrm{C}^{*}(G / \mathbb{T} ; G, \lambda)-\mathrm{C}^{*}(H / \mathbb{\pi} ; H, \beta)$-equivalence bimodule (see [22, Proposition 4.2]). This completes the proof.

Remark 3.3. Since $A_{1}$ and $B_{1}$ may be viewed as subalgebras of $A$ and $B$, respectively, all the proof of Theorem 3.2 amounts to is showing that $\rho_{Z}$ extends to a bounded linear operator on the completion of $C_{c}(Z)$ (with respect to the $A$ or $B$-valued inner product) in the sense of Rieffel [21] and is a self-adjoint projection there, mapping onto the closure of $C_{c}^{T}(Z)$.

Suppose that $H$ is a locally compact groupoid and that $Z$ is a locally compact, principal right $H$-space. Give $Z * Z=\left\{\left(z_{1}, z_{2}\right): \sigma\left(z_{1}\right)=\sigma\left(z_{2}\right)\right\}$ the diagonal action of $H$ (that is, $\left(z_{1}, z_{2}\right) \cdot \eta=\left(z_{1} \cdot \eta, z_{2} \cdot \eta\right)$, for $\left.\left(\left(z_{1}, z_{2}\right), \eta\right) \in(Z * Z) * H\right)$ and let $G=(Z * Z) / H$ be the quotient space. In [9] it is shown how to give $G$ the structure of a groupoid with unit space $Z / H$. Only the algebraic operations are checked there, but here we need to know that $G$ is a locally compact groupoid,

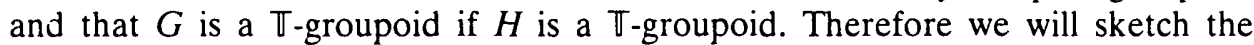
details for the sake of completeness. In the sequel, $G$ will be called the imprimitivity groupoid determined by the principal $H$-space $Z$.

Certainly $Z * Z$ is a locally compact space, since it is closed in $Z \times Z$. Furthermore, $G$ is the image of $Z * Z$ by a continuous open surjection (Lemma 2.1). Therefore $G$ is a locally compact space. Inversion is evidently a homeomorphism: $\left[z_{1}, z_{2}\right]^{-1}=\left[z_{2}, z_{1}\right]$. To show that multiplication is continuous, we may make systematic application of Lemma 2.1 and the following observation. Let $f: X \rightarrow Z$ and $g: Y \rightarrow Z$ be continuous maps (with $X, Y$, and $Z$, for the moment, arbitrary). Let $X * Y=\{(x, y) \in X \times Y: f(x)=g(y)\}$ have the relative topology. Consider the commutative diagram:

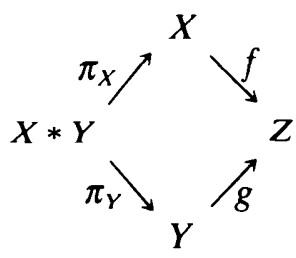

We proved on p. 7 of [9] that if $f$ is open, then so is $\pi_{Y}$. We shall call this the open-factor lemma.

To prove that multiplication is continuous, first observe that the unit space of $G$ may be identified with $Z / H$ and the range map is $r\left(\left[z_{1}, z_{2}\right]\right)=\left[z_{1}\right]$. We claim that $r$ is continuous and open. Indeed, consider the commutative diagram

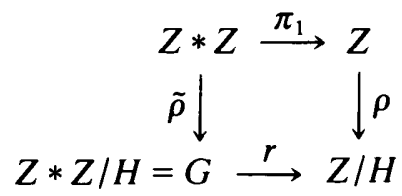

where $\pi_{1}$ is the projection onto the first factor and $\rho$ and $\tilde{\rho}$ denote the quotient maps for the $H$ actions. Since $Z * Z$ is the fibred product over the continuous, open map $\sigma, \pi_{1}$ is open by the open-factor lemma. It is clearly continuous. On the other hand, $\rho$ and $\tilde{\rho}$ are continuous and open by Lemma 2.1. Hence $r$ is open by a straightforward diagram chase. Of course the source map $s: G \rightarrow Z / H$, given by 
$s\left(\left[z_{1}, z_{2}\right]\right)=\left[z_{2}\right]$, is also continuous and open, since it is the composition of $r$ with inversion.

Consider, now, the commutative diagram

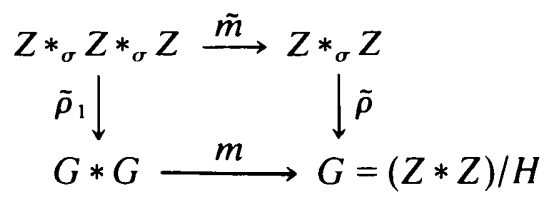

where $\tilde{\rho}$ is the quotient map, $\tilde{m}\left(z_{1}, z_{2}, z_{3}\right)=\left(z_{1}, z_{3}\right), m$ is multiplication, and $\tilde{\rho}_{1}\left(z_{1}, z_{2}, z_{3}\right)=\left(\left[z_{1}, z_{2}\right],\left[z_{2}, z_{3}\right]\right)$. Since $\tilde{\rho}$ and $\tilde{m}$ are continuous, to show that $m$ is continuous, it suffices to prove that the range of $\tilde{\rho}_{1}$ is all of $G * G$ and that $\tilde{\rho}_{1}$ is open. To verify the first statement, observe that $\left(\left[z_{1}, z_{2}\right],\left[z_{2}^{\prime}, z_{3}\right]\right) \in G * G$ if and only if $\left[z_{2}\right]=\left[z_{2}^{\prime}\right]$. This occurs exactly when there is an $\eta \in H$ such that $z_{2}^{\prime}=z_{2} \cdot \eta$. But $\left[z_{2} \cdot \eta, z_{3}\right]=\left[z_{2}, z_{3} \cdot \eta^{-1}\right]$ and then $\left(\left[z_{1}, z_{2}\right],\left[z_{2}^{\prime}, z_{3}\right]\right)=\tilde{\rho}_{1}\left(z_{1}, z_{2}, z_{3} \cdot \eta^{-1}\right)$. Thus, $\tilde{\rho}_{1}$ is surjective.

To show that $\tilde{\rho}_{1}$ is open, we use the fact that the quotient map from $Z * Z$ to $G$ is open (Lemma 2.1). Also notice that if $z_{\alpha} \rightarrow z$ and if $\left[z_{\alpha}, w_{\alpha}\right] \rightarrow[z, w]$, then $w_{\alpha}$ must converge to $w$ ! This follows from the fact that the $H$ action is free and proper. We can write any convergent net in $G * G$ in the form $\left(\left[z_{1}^{\alpha}, z_{2}^{\alpha}\right],\left[z_{2}^{\alpha}, z_{3}^{\alpha}\right]\right) \rightarrow\left(\left[z_{1}, z_{2}\right],\left[z_{2}, z_{3}\right]\right)$. As usual, we want to show that this net has a subnet which lifts to $Z *_{\sigma} Z *_{\sigma} Z$. Replacing $z_{i}^{\alpha}$ by $z_{i}^{\alpha} \cdot \eta_{\alpha}$, we may assume that $z_{1}^{\alpha} \rightarrow z_{1}$ and that $z_{2}^{\alpha} \rightarrow z_{2}$. The observation above shows that we must also have $z_{3}^{\alpha} \rightarrow z_{3}$, and $\tilde{\rho}_{1}$ is open onto its range, $G * G$. This completes that proof that $G$ is a locally compact groupoid.

Next observe that $G$ acts on the left of $Z$ as follows. First,

$$
G * Z=\left\{\left(\left[z_{1}, z_{2}\right], z_{3}\right):\left[z_{2}\right]=\rho\left(z_{3}\right)\left(=\left[z_{3}\right]\right)\right\} .
$$

Therefore given $\left(\left[z_{1}, z_{2}\right], z_{3}\right)$, there is an $\eta \in H$ such that $z_{2} \cdot \eta=z_{3}$. This $\eta$ is unique, since $H$ acts freely, and so, if we define $\left[z_{1}, z_{2}\right] \cdot z_{3}=z_{1} \cdot \eta$, then $\left[z_{1}, z_{2}\right] \cdot z_{3}$ is well defined. A moment's reflection reveals that this yields a bona fide free left $G$-action on $Z$.

\section{LEMMA 3.4. The action of $G$ on $Z$ is continuous and proper.}

Proof. Let $\Phi: Z * H \rightarrow Z \times Z$ be defined by $\Phi(x, \eta)=(z, z \cdot \eta)$. Recall that $\Phi$ is one-to-one and proper since $Z$ is a free and proper $H$-space. In fact, $\Phi$ is a homeomorphism onto a closed set: $Z *_{\rho} Z$ (Lemma 2.2). It follows that the map $\delta: Z *_{\rho} Z \rightarrow H$, defined by $\delta(z, z \cdot \eta)=\eta$, is continuous since it is the composition of $\Phi^{-1}$ followed by projection onto the second factor. The continuity of the $G$ action now follows from considering the commutative diagram

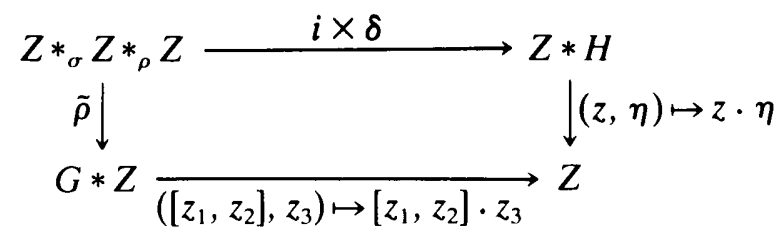

The right vertical map is continuous by assumption and we have just shown that $i \times \delta$ is continuous. Since $\tilde{\rho}$ is open by Lemma 2.1 , so is $\tilde{\rho} \times i$, and the continuity 
of the $G$-action, the lower horizontal map, follows. Since the $G$-action is free, to show that it is proper, we only need to show that the map $\Psi: G * Z \rightarrow Z \times Z$, given by $\Psi(\gamma, z)=(z, \gamma \cdot z)$, is closed. So suppose $\left\{\gamma_{\alpha}\right\}$ and $\left\{z_{\alpha}\right\}$ are nets such that the latter converges to $z$ and $\left\{\gamma_{\alpha} \cdot z_{\alpha}\right\}$ converges to $w$. We want to show that $\left\{\gamma_{\alpha}\right\}$ converges to $\gamma$ with $w=\gamma \cdot z$. But by definition of $G$ and the action, we may write each $\gamma_{\alpha}$ as $\left[x_{\alpha}, z_{\alpha}\right]$ with $\left[x_{\alpha}, z_{\alpha}\right] \cdot z_{\alpha}=x_{\alpha}$. By hypothesis, $\left\{z_{\alpha}\right\}$ converges to $z$ and $\left\{x_{\alpha}\right\}$ converges to $w$. Therefore $\left\{\left[x_{\alpha}, z_{\alpha}\right]\right\}=\left\{\gamma_{\alpha}\right\}$ converges to $\gamma:=[w, z]$ and $w=\gamma \cdot z$.

One checks easily, now, that $Z$ is a $(G, H)$-equivalence. If $H$ is also a

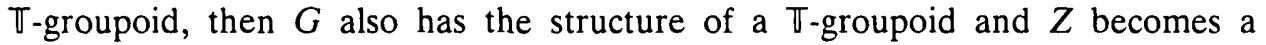

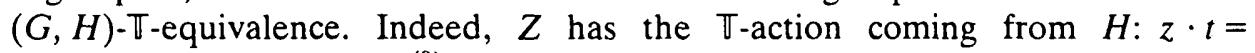
$z \cdot i(\sigma(z), t)$, where $i_{H}: H^{(0)} \times \mathrm{T} \rightarrow H$ is the embedding giving the $\mathrm{T}$-action on $H$. On the other hand, the map $i_{G}: Z / H \times \mathbb{T} \rightarrow G$ defined by the equation $i_{G}([z], t)=[z \cdot t, z]$ is easily seen to be well defined, by virtue of the fact that $H$ is

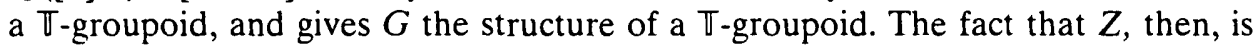

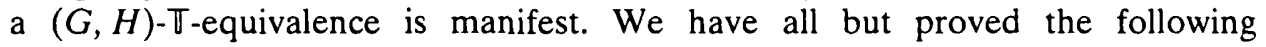
theorem.

THEOREM 3.5. Suppose that $H$ is a locally compact groupoid acting freely and properly on the right of a locally compact space $Z$.

(1) The imprimitivity groupoid $G=(Z * Z) / H$ is a locally compact groupoid acting freely and properly on the left of $Z$, and $Z$ is a $(G, H)$-equivalence.

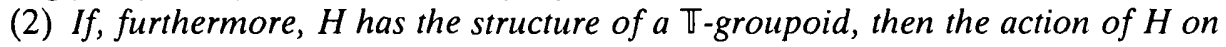
$Z$ gives $Z$ and $G \mathbb{T}$-actions in such a way as to convert $G$ into $a \mathbb{T}$-groupoid and $Z$ into a $(G, H)-\pi$-equivalence.

(3) If, in addition, $H / \mathbb{T}$ is a proper principal groupoid, then $G / T$ is also a proper principal groupoid and $G$ is a $\mathbb{T}$-groupoid over the relation $R_{G}$ in $Z / H \times Z / H$ given by $\left\{\left(r_{G}(\gamma), s_{G}(\gamma)\right): \gamma \in G\right\}$.

Proof. We have proved (1) and (2) above. In view of the above, to prove (3), it will suffice to prove that $j_{G}$, defined by $j_{G}\left(\left[z_{1}, z_{2}\right]\right)=\left(\left[z_{1}\right],\left[z_{2}\right]\right)$, is continuous, open, has closed range, and kernel $i_{G}(Z / H \times \mathbb{T})$ [11, Lemma 2.1]. By assumption, $j_{H}(\eta)=\left(r_{H}(\eta), s_{H}(\eta)\right)$ defines a continuous, open map of $H$ onto a closed subset $R_{H}$ of $H^{(0)} \times H^{(0)}$. It follows immediately that $j_{G}$ has kernel $i_{G}(Z / H \times \mathbb{T})$, and it is clearly continuous. Now suppose that $j_{G}\left(\left[z_{1}^{\alpha}, z_{2}^{\alpha}\right]\right) \rightarrow\left(\left[z_{1}\right],\left[z_{2}\right]\right)$. That is, $\sigma\left(z_{1}^{\alpha}\right)=$ $\sigma\left(z_{2}^{\alpha}\right),\left[z_{1}^{\alpha}\right] \rightarrow\left[z_{1}\right]$, and $\left[z_{2}^{\alpha}\right] \rightarrow\left[z_{2}\right]$. We may as well assume that $z_{1}^{\alpha} \rightarrow z_{1}$ and that there are $\eta_{\alpha} \in H$ such that $z_{2}^{\alpha} \cdot \eta_{\alpha} \rightarrow z_{2}$. Notice that $\left(\sigma\left(z_{1}^{\alpha}\right), \sigma\left(z_{2}^{\alpha} \cdot \eta_{\alpha}\right)\right) \in R_{H}$, and that $\left(\sigma\left(z_{1}^{\alpha}\right), \sigma\left(z_{2}^{\alpha} \cdot \eta_{\alpha}\right)\right) \rightarrow\left(\sigma\left(z_{1}\right), \sigma\left(z_{2}\right)\right)$. It follows that $\left(\sigma\left(z_{1}\right), \sigma\left(z_{2}\right)\right) \in R_{H}$. Moreover, since $j_{H}$ is open, we can pass to a subnet, relabel, and assume that there are $\beta_{\alpha} \rightarrow \beta$ in $H$ so that $r\left(\beta_{\alpha}\right)=\sigma\left(z_{1}^{\alpha}\right)$ and $r\left(\beta_{\alpha}\right)=\sigma\left(z_{1}^{\alpha} \cdot \eta_{\alpha}\right)$. We then have $z_{2}^{\alpha} \cdot \eta_{\alpha} \beta_{\alpha} \rightarrow z_{2} \cdot \beta$, and $\sigma\left(z_{1}\right)=s(\beta)=\sigma\left(z_{2} \cdot \beta\right)$. In particular, $j_{G}\left(\left[z_{1}, z_{2} \cdot \beta\right]\right)=\left(\left[z_{1}\right],\left[z_{2}\right]\right)$; this shows that $j_{G}$ has closed range. But $j_{G}\left(\left[z_{1}^{\alpha}, z_{2}^{\alpha} \cdot \eta_{\alpha} \beta_{\alpha}\right]\right)=j_{G}\left(\left[z_{1}^{\alpha}, z_{2}^{\alpha}\right]\right)$, so we have also shown that a convergent net in the range of $j_{G}$ has a subnet which lifts. This implies that $j_{G}$ is open. This completes the proof of (3).

REMARK 3.6. Of course if $G$ is a locally compact groupoid acting freely and properly on the left of a locally compact space $Z$, then there is an imprimitivity 
groupoid $H=G \backslash Z * Z$ and the conclusions of Theorem 3.5 hold with appropriate modifications.

REMARK 3.7. In the examples we know, a Haar system on $H$ will induce a Haar system on $G$, in fact, lots of them. However, we do not know the full story at this time. Fortunately, that will be of no concern to us here because, first of all, one of the rather attractive features of Theorem 3.2 is that it is independent of the choice of Haar system, and secondly, in our applications here, Haar systems on the imprimitivity groupoids will appear automatically.

\section{Topological results}

In this section, we attend to the exactness of the sequence in Theorem 1.1 at the first three points. The arguments are essentially those of Kumjian [6], but we want to take a slightly different perspective to that presented in [6], and we want to provide details for the reader's convenience and for the sake of completeness.

Exactness at $H^{1}(R, \mathbb{T})$. As mentioned in the introduction, we want to identify elements in $H^{1}(X / R, \mathscr{S})$ with (isomorphism classes of) circle bundles over $X / R$, and similarly for $H^{1}(X, \mathscr{S})$. To define a map $\eta: H^{1}(R, \mathbb{T}) \rightarrow H^{1}(X / R, \mathscr{S})$, we begin with a cocycle $c \in Z^{1}(R, \mathbb{T})$ and use it to define an action of $R$ on $X \times \mathbb{T}:(X \times \mathbb{J}) * R=\{((x, t),(x, y)):(x, y) \in R\}$ and $(x, t) \cdot(x, y)=(y, c(x, y) t)$. It is straightforward to verify that this action is free and proper. Thus the quotient space $S_{c}:=(X \times \mathbb{T}) / R$ is locally compact and Hausdorff. Moreover, the quotient map $q: X \times \mathbb{T} \rightarrow S_{c}$ is open by Lemma 2.1. We write $[x, t]$ for $q(x, t)$. The action of $\mathbb{T}$ on $X \times \mathbb{T}$ determined by translation in the second variable commutes with the action of $R$ and therefore gives rise to a free, continuous action of $\mathbb{T}$ on $S_{c}: s \cdot[x, t]=[x, s t]$. Furthermore, it is clear that set-theoretically $\mathbb{T} \backslash S_{c}$ is $X / R$ and

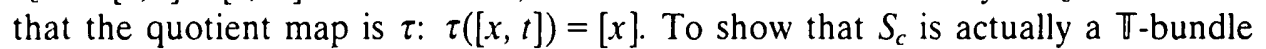
we need only show that $\tau$ is continuous and open. However, this is obvious for the following commutative diagram:

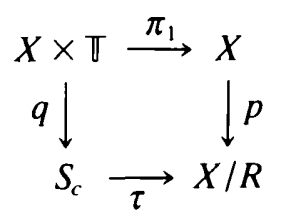

Indeed, the vertical arrows are the quotient maps determined by $R$-actions and so are continuous and open surjections by Lemma 2.1. The map $\pi_{1}$ is projection onto the first factor and is therefore a continuous and open surjection as well. Thus $\tau$ is also continuous and open.

Suppose that $c_{1}$ and $c$ are cohomologous cocycles with $c_{1}(x, y)=$ $b(x) \overline{b(y)} c(x, y)$ for a continuous map $b: X \rightarrow \mathbb{T}$, and define $\Phi: X \times \mathbb{T} \rightarrow X \times \mathbb{T}$ by $\Phi(x, t)=(x, \overline{b(x)} t)$. Then $\Phi$ is a homeomorphism that intertwines the two $R$ actions determined by $c$ and $c_{1}$ and is equivariant with respect to the $\mathbb{T}$-action on $X \times \mathrm{T}$. It follows that $\Phi$ implements a bundle isomorphism between $S_{c}$ and $S_{c_{1}}$. Thus $\eta$ may be viewed as a map from $H^{1}(R, \mathbb{T})$ to $H^{1}(X / R, \mathscr{S})$. Moreover, what we have just seen shows that if $c$ is a coboundary, then $S_{c}$ is the trivial bundle. 
But if $S_{c}$ is trivial, then there is a bundle isomorphism $\phi: S_{c} \rightarrow X / R \times \mathbb{T}$ which determines a continuous $\mathbb{T}$-valued map $h$ on $X$ through the composition

$$
x \longmapsto(x, 1) \stackrel{q}{\longmapsto}[x, 1] \stackrel{\phi}{\longmapsto}([x], h(x)) \longmapsto h(x) .
$$

If $y$ is equivalent to $x$, modulo $R$, then we have

$$
\begin{aligned}
([x], h(y)) & =([y], h(y))=\phi([y, 1]) \\
& =\phi([x, \overline{c(x, y)}])=\overline{c(x, y)} \phi([x, 1]) \\
& =\overline{c(x, y)}([x], h(x))=([x], \overline{c(x, y)} h(x)),
\end{aligned}
$$

which shows that $c$ is the coboundary determined by $h$. Thus, if we show that $\eta$ is a homomorphism, then it will be injective.

To this end, consider the bundles $S_{c}$ and $S_{d}$ determined by two cocycles $c$ and $d$. Their product $S_{c} \cdot S_{d}$ is formed by first forming the fibred product $S_{c} * S_{d}=$ $\left\{([x, t],[y, s]) \in S_{c} \times S_{d}:[x]=[y]\right\}$ and taking the quotient by the diagonal action of $\mathbb{T}: s \cdot\left(\left[x, t_{1}\right],\left[y, t_{2}\right]\right)=\left(\left[x, s^{-1} t_{1}\right],\left[y, s t_{2}\right]\right)$. Elements in this quotient will be denoted $[[x, t],[y, s]]$. Note that for $r \in \mathbb{T}$ we have $[[x, t r],[y, s]]=[[x, t],[y, s r]]$ and that there is a natural $\mathbb{T}$-action given by $r \cdot[[x, t],[y, s]]=[[x, r t],[y, s]]$. One

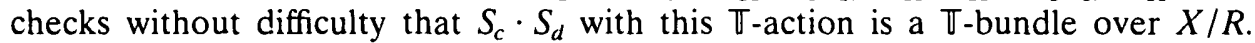
Define a map from $X \times \mathrm{T}$ to $S_{c} \cdot S_{d}$ by sending $(x, t)$ to $[[x, t],[x, 1]]$ and observe that this map passes to the quotient $S_{c d}$. Indeed, $(x, t)$ and $(y, s)$ are identified in this quotient if and only if $(x, y) \in R$ and $s=c(x, y) d(x, y) t$. In this event, we have

$$
\begin{aligned}
{[[y, c(x, y) d(x, y) t],[y, 1]] } & =[[x, d(x, y) t],[y, 1]] \\
& =[[x, t],[y, d(x, y) 1]]=[[x, t],[x, 1]] .
\end{aligned}
$$

It is not hard to see, now, that this map implements a bundle isomorphism between $S_{c d}$ and $S_{c} \cdot S_{d}$. This shows that our sequence is exact at $H^{1}(R, \mathbb{T})$.

Exactness at $H^{1}(X / R, \mathscr{S})$. If one views elements of $H^{1}(X / R, \mathscr{S})$ and $H^{1}(X, \mathscr{S})$ as (isomorphism classes of) bundles, then the map $p^{*}$ is the usual pull-back: if $\tau: S \rightarrow X / R$ is a $\mathbb{T}$-bundle, then $p^{*}(S)=\{(x, \xi): \tau(\xi)=p(x)\}$ and the bundle map, $\tau^{*}$, is projection onto the first factor. If $S$ is in the range of $\eta$, then we may assume that $S=S_{c}$ for a suitable cocycle $c$. The map $\sigma: X \rightarrow p^{*}\left(S_{c}\right)$ defined by $\sigma(x)=(x,[x, 1])$ is a continuous section for $\tau^{*}$, and so $p^{*}\left(S_{c}\right)$ is trivial and $\operatorname{Im}(\eta) \subseteq \operatorname{ker}\left(p^{*}\right)$

For the reverse inclusion, suppose that $S \in H^{1}(X / R, \mathscr{S})$ is such that $p^{*}(S)$ is trivial. There is, then, a continuous section $\tilde{\sigma}: X \rightarrow p^{*}(S)$ (that is, $\tau^{*} \tilde{\sigma}(x)=x$ ). Using the definition of $\tau^{*}$, we see that there must be a continuous map $\sigma: X \rightarrow S$ such that $\tilde{\sigma}(x)=(x, \sigma(x))$ in $p^{*}(S)$. This means, in particular, that $\tau(\sigma(x))=[x]$. So, if $(x, y) \in R$, then $\tau(\sigma(x))=\tau(\sigma(y))$. By virtue of the freeness of the $\mathbb{T}$-action on $S$, there is a unique $c(x, y) \in \mathbb{T}$ such that $\sigma(y)=c(x, y) \sigma(x)$. The continuity of

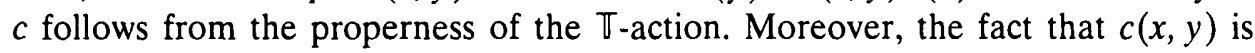
uniquely determined by $(\sigma(x), \sigma(y))$, when $(x, y) \in R$, and the fact that $S$ is a

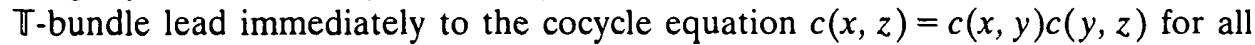
$(x, y),(y, z) \in R$. To see that $S$ is isomorphic to $S_{c}$, simply observe that the map

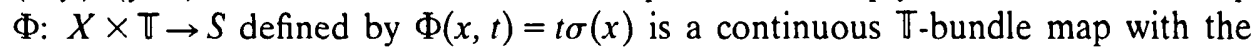
property that $\Phi(x, t)=\Phi(y, s)$ if and only if $[x]=[y]$ and $t=c(x, y) s$; that is, if 
and only if $[x, t]=[y, s]$ in $S_{c}$. Thus $\Phi$ passes to a bijection between $S_{c}$ and $S$,

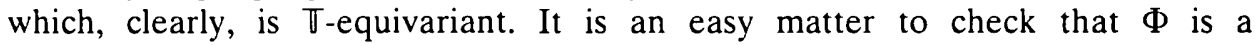
homeomorphism and we conclude, therefore, that $S$ and $S_{c}$ are isomorphic. This shows that $\operatorname{ker}\left(p^{*}\right) \subseteq \operatorname{Im}(\eta)$ and completes the proof of exactness at $H^{1}(X / R, \mathscr{P})$.

Exactness at $H^{1}(X, \mathscr{S})$. To show exactness at $H^{1}(X, \mathscr{S})$, we need first to comment on how the map $\varepsilon: H^{1}(X, S) \rightarrow \operatorname{Tw}(R)$ is defined. Given a bundle $\tau: S \rightarrow X$, we may view $S$ as a right $H$-space over $H=\mathbb{T}$ viewed as a $\mathbb{T}$-groupoid. Evidently, $S$ with this action of $H$ is a free and proper $H$-space. By Theorem 3.5, the imprimitivity groupoid $S * S / H$ has the structure of a T-groupoid, $\tilde{G}_{\tau}$, and $S$ is a $\left(\widetilde{G}_{\tau}, H\right)$ - $\mathbb{T}$-equivalence. One checks easily that the underlying relation of $\widetilde{G}_{\tau}$, that is, the base of $\widetilde{G}_{\tau}$ as a T-bundle, is all of $X \times X$. We let $G_{\tau}$ be the restriction (as a T-bundle) of $\tilde{G}_{\tau}$ to $R$, and we define $\varepsilon(S)$, or $\varepsilon(\tau)$, to be $G_{\tau}$.

To see that $\varepsilon$ is a homomorphism, recall how the product of two $\mathbb{T}$-bundles and the product of two T-groupoids are defined. Suppose that for $i=1,2, \tau_{i}: S_{i} \rightarrow X$ is

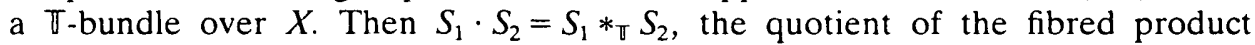
$S_{1} * S_{2}=\left\{\left(\xi_{1}, \xi_{2}\right): \tau_{1}\left(\xi_{1}\right)=\tau_{2}\left(\xi_{2}\right)\right\}$ by the diagonal T-action, $t \cdot\left(\xi_{1}, \xi_{2}\right)=$ $\left(t \cdot \xi_{1}, t^{-1} \cdot \xi_{2}\right)$. The bundle map $\tau_{1} \cdot \tau_{2}: S_{1} \cdot S_{2} \rightarrow X$ is given by the formula $\tau_{1} \cdot \tau_{2}\left(\left[\xi_{1}, \xi_{2}\right]\right)=\tau_{1}\left(\xi_{1}\right)$. Likewise for $\mathbb{T}$-groupoids $j_{i}: G_{i} \rightarrow R(i=1,2), G_{1} \cdot G_{2}$ is simply the product bundle endowed with the obvious groupoid product: $\left[\alpha_{1}, \alpha_{2}\right]$ and $\left[\beta_{1}, \beta_{2}\right]$ are composable if and only if $\alpha_{1}$ and $\beta_{1}$ are (which implies $\alpha_{2}$ and $\beta_{2}$ are as well) in which case $\left[\alpha_{1}, \alpha_{2}\right]\left[\beta_{1}, \beta_{2}\right]=\left[\alpha_{1} \beta_{1}, \alpha_{2} \beta_{2}\right]$. A moment's reflection reveals that this product is well defined and that with inversion defined by $\left[\alpha_{1}, \alpha_{2}\right]^{-1}=\left[\alpha_{1}^{-1}, \alpha_{2}^{-1}\right], G_{1} \cdot G_{2}$ becomes a T-groupoid over $R$. Moreover, with respect to this product and the process of inversion, which assigns to a $\mathbb{T}$-groupoid $G$ the conjugate $\mathbb{T}$-action $t \cdot \gamma=\bar{t} \cdot \gamma$, the collection of $\mathbb{T}$-groupoids over $R$ form a group with identity $R \times \mathbb{T}$ (with the obvious groupoid structure). This group is, essentially, what we are calling $\operatorname{Tw}(R)$ and which was defined earlier in terms of extensions. Strictly speaking, we should by identifying isomorphic $\mathbb{T}$-groupoids, but the lapse in precision should cause no harm.

So, let $S_{1}$ and $S_{2}$ be two T-bundles over $X$. The map which sends

$$
\begin{aligned}
\left(\xi_{1}, \xi_{2}, \xi_{1}^{\prime}, \xi_{2}^{\prime}\right) \in\left(S_{1} * S_{2}\right) *\left(S_{1}\right. & \left.* S_{2}\right) \\
& =\left\{\left(\xi_{1}, \xi_{2}, \xi_{1}^{\prime}, \xi_{2}^{\prime}\right): \tau_{1}\left(\xi_{1}\right)=\tau_{2}\left(\xi_{2}\right), \tau_{1}\left(\xi_{1}^{\prime}\right)=\tau_{2}\left(\xi_{2}^{\prime}\right)\right\}
\end{aligned}
$$

to

$$
\begin{aligned}
\left(\xi_{1}, \xi_{1}^{\prime}, \xi_{2}, \xi_{2}^{\prime}\right) \in\left(S_{1} * S_{1}\right) *\left(S_{2} * S_{2}\right) & \\
& =\left\{\left(\xi_{1}, \xi_{1}^{\prime}, \xi_{2}, \xi_{2}^{\prime}\right): \tau_{1}\left(\xi_{1}\right)=\tau_{2}\left(\xi_{2}\right), \tau_{1}\left(\xi_{1}^{\prime}\right)=\tau_{2}\left(\xi_{2}^{\prime}\right)\right\}
\end{aligned}
$$

is a homeomorphism which is equivariant with respect to all the various $\mathbb{T}$ - and $X \times \mathbb{T}$-actions and gives rise to the commutative diagram

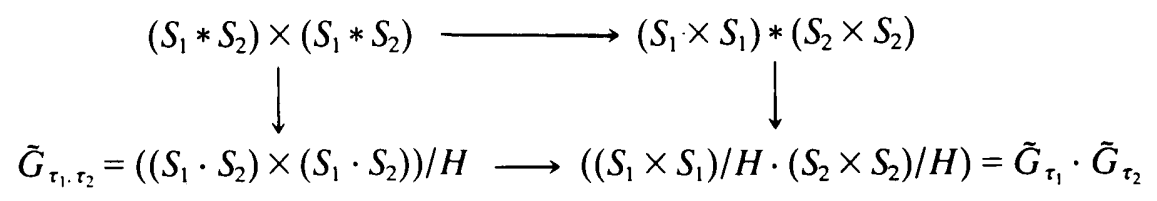

It follows easily that the lower horizontal map is an isomorphism and so, after 
restricting to $R$, we see that $G_{\tau_{1} \cdot \tau_{2}}$ is isomorphic to $G_{\tau_{1}} \cdot G_{\tau_{2}}$. This completes the proof that $\varepsilon$ is a homomorphism.

To identify the kernel of $\varepsilon$, first recall from Theorem 3.5 that given a $\mathbb{J}$-bundle $\tau: S \rightarrow X$, the groupoid $\tilde{G}_{\tau}$ acts freely and properly on $S$ by the formula $\left[s_{1}, s_{2}\right] \cdot s_{2}=s_{1}$. Restricting $\widetilde{G}_{\tau}$ to $R$ gives an action of $G_{\tau}$ on $S$, which is free and proper too. Suppose, now, that $G_{\tau}$ is trivial as a T-groupoid, meaning that $\varepsilon(S)=0$ and that $G_{\tau}$ is isomorphic to $R \times \mathbb{T}$. Then there is an injective groupoid homomorphism $\sigma: R \rightarrow G_{\tau}$ which is a cross section for the bundle map $j: G_{\tau} \rightarrow R$. It follows that the action of $R$ on $S$ defined by the formula $(x, y) \cdot s=\sigma(x, y) \cdot s, \tau(s)=y$, is free and proper and, moreover, it commutes with the $\mathbb{T}$-action on $S$ because $G_{\tau}$ is a $\mathbb{T}$-groupoid and $\sigma$ is a section. It follows, then, that $R \backslash S$ becomes a $\mathbb{T}$-bundle over $X / R$. Form the pull-back

$$
p^{*}(R \backslash S)=\{(x,[s]) \in X \times R \backslash S: p(x)=p(\tau(s))\}
$$

and define $\phi: p^{*}(R \backslash S) \rightarrow S$ by $\phi(x,[s])=\sigma(x, \tau(s)) \cdot s$. This is easily checked to be a well-defined bundle isomorphism from $p^{*}(R \backslash S)$ onto $S$. Thus, $\operatorname{ker}(\varepsilon) \subseteq$ $\operatorname{Im}\left(p^{*}\right)$.

As for the reverse inclusion, let $\widetilde{S}$ be a $\mathbb{T}$-bundle over $X / R$ and let $S=p^{*}(\widetilde{S})$

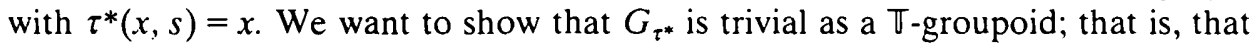
there is a homomorphism $\sigma: R \rightarrow G_{\tau^{*}}$ that is a cross section for $j: G_{\tau^{*}} \rightarrow R$. But this is easy. Given $(x, y) \in R$, choose $s \in \tilde{S}$ such that $\tau(s)=p(x)=p(y)$, and define $\sigma(x, y)=[(x, s),(y, s)]$ in $\widetilde{G}_{\tau^{*}}=S \times S / \mathbb{T}$. By definition of the $\mathbb{T}$-action, $[(x, s),(y, s)]$ is independent of the choice of $s$ satisfying $\tau(s)=p(x)$, and so $\sigma$ is a well-defined map from $R$ into $G_{\tau}$ and is a cross section for $j$. An easy computation shows that $\sigma$ is a homomorphism. The continuity is an easy consequence of the fact that $S \times\left. S\right|_{R}$ is a $\mathbb{T}^{2}$-bundle over $R$ and, therefore, that the quotient map is open.

\section{The Dixmier-Douady class of a restricted $\mathbb{T}$-groupoid $C^{*}$-algebra}

In this section we prove the exactness of the sequence in Theorem 1.1 at $\mathrm{Tw}(R)$. Recall that $R$ is a principal groupoid acting properly on its unit space $X$. We are assuming that $R$ has a Haar system, and we fix one, $\lambda$, for the rest of this paper. If $G$ is a $\mathrm{T}$-groupoid over $R$, then $\mathrm{C}^{*}(R ; G, \lambda)$ is a continuous-trace $C^{*}$-algebra with spectrum $X / R\left[\mathbf{1 2}\right.$, Theorem 4.2 and Proposition 3.3]. If $G^{\prime}$ is equivalent to $G$, that is, if $[G]=\left[G^{\prime}\right]$ in $\operatorname{Tw}(R)$, then $\mathrm{C}^{*}(R ; G, \lambda)$ is (strongly) Morita equivalent to $C^{*}\left(R ; G^{\prime}, \lambda\right)$, so we obtain a well-defined map $\delta: \operatorname{Tw}(R) \rightarrow$ $H^{2}(X / R, \mathscr{S})$ by defining $\delta([G])$ to be the Dixmier-Douady class $\delta\left(\mathrm{C}^{*}(R ; G, \lambda)\right)$. (There is no loss of generality in fixing $\lambda$. If $\lambda^{\prime}$ is another Haar system, then $G$ is a $(G, G)$ - -equivalence and $\mathrm{C}^{*}\left(R ; G, \lambda^{\prime}\right)$ and $\mathrm{C}^{*}(R ; G, \lambda)$ are (strongly) Morita equivalent by Theorem 3.2.)

The next step is to investigate $\delta\left(\mathrm{C}^{*}(R ; G, \lambda)\right)$ in view of our assumption that the quotient map $p: X \rightarrow X / R$ has local sections. As we intend to calculate $\delta\left(\mathrm{C}^{*}(R ; G, \lambda)\right)$ using the approach in [17], we must use the fact that $\mathrm{C}^{*}(R ; G, \lambda)$ is locally Morita equivalent to $C_{0}(X / R)$. Then $\delta\left(C^{*}(R ; G, \lambda)\right)$ will be the obstruction to $\mathrm{C}^{*}(R ; G, \lambda)$ being globally Morita equivalent to $C_{0}(X / R)$. Our local Morita equivalences will result from the following.

Lemma 5.1. Suppose that $G$ is a T-groupoid over a principal proper groupoid $R$ with Haar system $\lambda$. If the natural map $p: X \rightarrow X / R$ has a continuous section $\omega$, 
then $\rho=\left.r\right|_{G_{F}}$ is open. In particular, $\mathrm{C}^{*}(G, \tilde{\lambda})$ is (strongly) Morita equivalent to $C_{0}(X / R \times \mathbb{Z})$, and $\mathrm{C}^{*}(R ; G, \lambda)$ is (strongly) Morita equivalent to $C_{0}(X / R)$.

Proof. As in the proof of Corollary 2.6, $G$ will be equivalent to $G_{F}^{F}$, where $F$ is the transversal $\omega(X / R)$, provided $\rho$ is open. This will follow from Theorem 2.5 once we show that $\pi_{G}=(r, s): G \rightarrow X \times X$ is an open map onto its range. However, this is immediate since $\pi_{G}=\pi_{R} \circ j$, where $j$ is the bundle map from $G$ onto $R$ and $\pi_{R}$ is a homeomorphism onto its range [11, Lemma 2.1(ii)]. But $G_{F}^{F}$ is a $\mathbb{T}$-bundle (Corollary 5.4) which is trivial since $u \mapsto u$ is a section. Thus $G_{F}^{F}$ is homeomorphic to $F \times \mathbb{T} \cong X / R \times \mathbb{T}$. The desired result now follows from Theorem 3.2 .

REMARK 5.2. Recall that the bimodule implementing the equivalence between $A=C^{*}(R ; G, \lambda)$ and $B=C_{0}(X / R)$ is the completion of $C_{c}^{\pi}\left(G_{F}\right)$ with respect to the operations given in Equations (3.3), (3.4), (3.9), and (3.10). In this case these simplify to

$$
\begin{aligned}
f \cdot \phi(\gamma) & =\int_{R} f(\alpha) \phi\left(\alpha^{-1} \gamma\right) d \lambda^{r(\alpha)}(\dot{\alpha}), \\
\phi \cdot g(\gamma) & =\phi(\gamma) g(p(s(\gamma))), \\
{ }_{A}\langle\phi, \psi\rangle(\gamma) & =\phi(\gamma \eta) \overline{\psi(\eta)}, \quad \text { where } \eta \in G_{F} \text { and } r(\eta)=s(\gamma), \\
\langle\phi, \psi\rangle_{B}(\dot{u}) & =\int_{R} \overline{\phi\left(\gamma^{-1}\right)} \psi\left(\gamma^{-1}\right) d \lambda^{\omega(u)}(\dot{\gamma}) .
\end{aligned}
$$

Recall that if $S$ is a subset of $X$, then $[S]$ denotes the saturation of $S$ : $[S]=p^{-1}(p(S))$.

LEMma 5.3. Suppose that $F$ and $E$ are closed transversals arising from local sections in a $\mathbb{J}$-groupoid $G$ with open range and source maps $r$ and $s$. Let $q=\left.r\right|_{G \text { E. }}$. Then $q: G_{E}^{F} \rightarrow F$ is a principal T-bundle.

Proof. Since $G_{E}^{F}$ is clearly a $\backslash$-space, we merely have to see that $q$ identifies $G_{E}^{F} / \pi$ with $F$. First notice that $[F]=G^{(0)}=[E]$ implies $q$ is surjective. Furthermore, $\rho=\left.r\right|_{G_{E}}$ is open (Lemma 5.1) from $G_{E}$ onto $X$ and $G_{E}^{F}=\rho^{-1}(F)$. Thus $q$ is open. Finally, if $\gamma, \beta \in G_{E}^{F}$ and $r(\gamma)=r(\beta)$, then $s(\gamma) \sim s(\beta)$, and since $E$ is a transversal, $s(\gamma)=s(\beta)$. Thus $\gamma=t \cdot \beta$ for some $t \in \mathbb{T}$. The assertion follows.

Corollary 5.4. Suppose that $G$ is a $\mathbb{T}$-groupoid with open range and source maps $r$ and s. Also suppose that $F$ and $E$ are closed transversals corresponding to continuous sections $\omega$ and $\sigma$. Then each point in $X / G$ has a closed neighbourhood $C$ so that if $F^{\prime}=\omega(C)$ and $E^{\prime}=\sigma(C)$, then there is a continuous map $\phi: F^{\prime} \rightarrow G_{E^{\prime}}^{F^{\prime}}$ such that $r(\phi(u))=u$ and $s(\phi(u))=\sigma(p(u))$.

REMARK 5.5. Maps such as $\phi$ are essentially admissible sections in the sense of Mackenzie [8, Definition II.5.7].

Proof. By Lemma 5.3 and [5], $q: G_{E}^{F} \rightarrow F$ is locally trivial. Choose $C$ so that there is a continuous section $\phi: F^{\prime}=\omega(C) \rightarrow G_{E}^{F}$. By definition, $r(\phi(u))=u$ for 
all $u \in F^{\prime}$; thus $r\left(\phi\left(F^{\prime}\right)\right)=F^{\prime}$. On the other hand, $s(\phi(u)) \in E^{\prime}=\sigma(C) \subset E$ and $\sigma(p(u)) \sim u$. Since $E$ is a transversal, $s(\phi(u))=\sigma(p(u))$ and $\phi\left(F^{\prime}\right) \subseteq G_{E^{\prime}}^{F^{\prime}}$.

At this point, we need to fix some (considerable) notation. By assumption there is a collection $\left\{C_{i}\right\}_{i \in l}$ of closed neighbourhoods in $X / R=X / G$ whose interiors cover $X / R$, and for which there exist continuous sections $\omega_{i}: C_{i} \rightarrow X$. Let $F_{i}=\omega_{i}\left(C_{i}\right)$ and $F_{i j}=\omega_{i}\left(C_{i j}\right)$. Note that in general, $F_{i j} \neq F_{j i}$, although $F_{i j}$ and $F_{j i}$ are both transversals for $T=\left[F_{i j}\right]=\left[F_{i j}\right]$. The next argument shows that we can refine our covers to ensure that the right sort of maps are defined on all overlaps. The argument follows that of [3, Lemma 10.7.11].

Lemma 5.6. Suppose that $G$ is a T-groupoid over $R$ and that $R$ is a proper principal groupoid with Haar system $\lambda$ and local sections for $p: X \rightarrow X / R$. Then there are closed neighbourhoods $\left\{C_{i}\right\}_{i \in I}$ in $X / R$ whose interiors cover $X / R$ such that

(1) there are continuous sections $\omega_{i}: C_{i} \rightarrow X$, and

(2) there are continuous maps $\phi_{i j}: F_{i j} \rightarrow G_{F_{j i}}^{F_{i j}}$ such that $r\left(\phi_{i j}(u)\right)=u$ and $s\left(\phi_{i j}(u)\right)=\omega_{j}(p(u))$ for all $u \in F_{i j}$.

Here we use the notation developed above: $F_{i}=\omega_{i}\left(C_{i}\right)$ and $F_{i j}=\omega_{i}\left(C_{i j}\right)$.

Remark 5.7. Notice that if $\gamma \in G_{F_{i j}}$ then $\gamma \phi_{i j}(s(\gamma)) \in G_{F_{j i}}$ Thus, essentially, $\phi$ determines what Mackenzie [8] would call a local right translation, mapping $G_{F_{i j}}$ to $G_{F_{i i}}$. These are related to the notion of 'glissement à droite' of Albert and Dazord in [1] and to the notion of 'bisection' in [2].

Proof. We can certainly find a collection $\left\{T_{\lambda}\right\}_{\lambda \in A}$ of closed neighbourhoods, whose interiors $V_{\lambda}$ cover $X / R$, with the property that there are continuous sections $\omega_{\lambda}: T_{\lambda} \rightarrow X$. Since our assumptions imply that $X / R$ is Hausdorff and therefore paracompact, we can assume that $\left\{V_{\lambda}\right\}_{\lambda \in A}$ is locally finite; hence there is an open cover $\left\{U_{\lambda}\right\}_{\lambda \in A}$ satisfying $M_{\lambda}=\overline{U_{\lambda}} \subseteq V_{\lambda}$.

Now fix $\lambda \in A$ and $x \in U_{\lambda}$. By local finiteness, there is a neighbourhood $Z$ of $x$ such that

$$
A^{\prime}=\left\{\beta \in A: Z \cap M_{\beta} \neq \varnothing\right\}
$$

is finite. We claim that for each $\beta \in A$, there is a closed neighbourhood $Z_{\beta}$ of $x$ such that $Z_{\beta} \subseteq Z$ and such that there is a continuous function $\psi: \omega_{\lambda}\left(Z_{\beta} \cap M_{\beta}\right) \rightarrow$ $G_{\boldsymbol{w}_{\beta}}^{\omega_{\lambda}\left(Z_{\beta} \cap Z_{\beta} \cap M_{\beta}\right)}$ satisfying (2). If $x \notin M_{\beta}$, then we can simply choose $Z_{\beta}$ such that $Z_{\beta} \cap M_{\beta}=\varnothing$. If $x \in M_{\beta}$, then we can apply Corollary 5.4 to the restriction of $G$ to $T_{\gamma} \cap T_{\beta}$. The point is that $Z_{\beta, x}=\bigcap_{\beta \in A^{\prime}} Z_{\beta}$ is a closed neighbourhood of $x$ with the property that there is a function from $\omega_{\gamma}\left(Z_{\gamma, x} \cap M_{\beta}\right)$ to $G_{\omega_{\beta}\left(Z_{\gamma, x} \cap M_{\beta}\right)}^{\omega_{\gamma}\left(Z_{\gamma, x} \cap M_{\beta}\right)}$ satisfying (2) for all $\beta \in A$.

The desired cover is obtained by letting $I=\left\{(\gamma, x) \in A \times X / R: x \in U_{\gamma}\right\}$. For each $i \in I$, we let $C_{i}=W_{i}$. 
Next define $h_{i j}: G_{F_{i j}} \rightarrow G_{F_{j i}}$ by $h_{i j}(\gamma)=\gamma \phi_{i j}(s(\gamma))$. Each $h_{i j}$ implements a T-bundle equivalence:

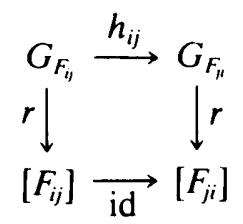

There are several things to check here. The first is that $G_{F_{i j}} \rightarrow\left[F_{i j}\right]$ is really a $\pi$-bundle; this follows from Theorem 2.5 applied to the restriction of $G$ to $\left[F_{i j}\right]$. Since $h_{i j}$ is certainly continuous and takes values in $G_{F_{j i}}$ (Remark 5.7), and the diagram commutes, the rest follows from [13, Lemma 1.12].

Define $\theta_{i j}: C_{c}\left(G_{F_{j i}}\right) \rightarrow C_{c}\left(G_{F_{i j}}\right)$ by $\theta_{i j}(f)(\gamma)=f\left(h_{i j}(\gamma)\right)=f\left(\gamma \phi_{i j}(s(\gamma))\right)$. Now if $F_{i j k}=\omega_{i}\left(C_{i j k}\right), \gamma \in G_{F_{i j k}}$, and $[u]=p(s(\gamma)) \in C_{i j k}$, then

$$
\begin{aligned}
\theta_{i j} \circ \theta_{j k}(f)(\gamma) & =\theta_{j k}(f)\left(\gamma \phi_{i j}(s(\gamma))\right) \\
& =f\left(\gamma \phi_{i j}(s(\gamma)) \phi_{j k}\left(s\left(\phi_{i j}(s(\gamma))\right)\right)\right) \\
& =f\left(\gamma \phi_{i j}\left(\omega_{i}([u])\right) \phi_{j k}\left(\omega_{j}([u])\right)\right) .
\end{aligned}
$$

On the other hand, $\theta_{i k}(f)(\gamma)=f\left(\gamma \phi_{i k}\left(\omega_{i}([u])\right)\right)$. By Lemma 5.1, $\mathrm{C}^{*}\left(\left.R\right|_{\left[F_{i}\right]} ;\left.G\right|_{\left[F_{i}\right]}, \lambda\right)$ is (strongly) Morita equivalent to $C_{0}\left(C_{i}\right)$. Thus $\mathrm{C}^{*}(R ; G, \lambda)$ is locally Morita equivalent to $C_{0}(X / R)$ (cf. [17, §3]). Using the notation in the discussion following $\left[\mathbf{1 7}\right.$, Lemma 3.4], we let $\mathfrak{X}_{i}$ be the $C^{*}\left(\left.R\right|_{\left[F_{i}\right]} ;\left.G\right|_{\left[F_{i}\right]}, \lambda\right)-C_{0}\left(C_{i}\right)$ imprimitivity bimodule which is the completion fo $C_{c}^{\mathbb{\pi}}\left(G_{F_{1}}\right)$ with respect to the operations (5.1)-(5.4). Then $\mathfrak{X}_{i}^{C_{i j}}$ may be identified with the completion of those functions in $C_{c}^{\mathbb{\pi}}\left(G_{F_{1}}\right)$ which vanish off $\left\{\gamma \in G_{F_{i}}: s(\gamma) \in p^{-1}\left(C_{j}\right)\right\}$. Thus $\mathfrak{X}_{i}^{C_{i j}}$ is the completion of $C_{c}^{\top}\left(G_{F_{i}}\right)$ with respect to the operations (5.1)-(5.4). The point is that the $\theta_{i j}$ respect the operations $(5.1)-(5.4)$, and so define imprimitivity bimodule isomorphisms $\widetilde{\theta}_{i j}: \mathfrak{X}_{j}^{C_{i j}} \rightarrow \mathfrak{X}_{i}^{C_{i j}}$. Thus it follows from $[17$, discussion following Lemma 3.4 and Lemma 6.2], that there is a 2-cocycle $v=\left\{v_{i j k}\right\}$ in $H^{2}\left(\left\{C_{i}\right\}, \mathscr{S}\right)$ defined by

$$
\tilde{\theta}_{i j} \circ \tilde{\theta}_{j k}=v_{i j k} \tilde{\theta}_{i k}
$$

such that $\delta\left(\mathrm{C}^{*}(R ; G, \lambda)\right)=[v]$. On the other hand,

$$
\gamma=\phi_{i j}\left(\omega_{i}([u])\right) \phi_{j k}\left(\omega_{j}([u])\right) \phi_{i k}\left(\omega_{i}([u])\right)^{-1}
$$

satisfies $r(\gamma)=\omega_{i}([u])=s(\gamma)$. Consequently (here, we identify $X \times \mathbb{T}$ with a subset of $G$ ),

$$
\gamma=\left(\omega_{i}([u]), v_{i j k}([u])\right)
$$

If $\left[G^{\prime}\right] \in \operatorname{Tw}(R)$ as well, then, since we can take refinements at will, we may assume that for the same cover $\left\{C_{i}\right\}$, we have sections $\omega_{i}^{\prime}$ and produce the corresponding data $F_{i}^{\prime}, \phi_{i j}^{\prime}, h_{i j}^{\prime}, \theta_{i j}^{\prime}$, and $v_{i j k}^{\prime}$. But then it is immediate that we obtain sections $\omega^{\prime \prime}$ for $G * G^{\prime}$ on $\left\{C_{i}\right\}$ by defining $\omega_{i}^{\prime \prime}(c)$ to be the class $\left[\omega_{i}(c), \omega_{i}^{\prime}(c)\right]$. The corresponding data $F_{i}^{\prime \prime}, \phi_{i j}^{\prime \prime}, h_{i j}^{\prime \prime}, \theta_{i j}^{\prime \prime}$, and $v_{i j}^{\prime \prime}$ are now easily computed in terms of the previous data. In fact,

$$
\tilde{\theta}_{i j}^{\prime \prime} \circ \tilde{\theta}_{j k}^{\prime \prime}=v_{i j k} v_{i j k}^{\prime} \tilde{\theta}_{i k}^{\prime \prime} \text {. }
$$

In other words, $\delta: \operatorname{Tw}(R) \rightarrow H^{2}(X / R, \mathscr{S})$ is a homomorphism. 
Exactness at $\operatorname{Tw}(R)$. Now suppose that $\delta\left(C^{*}(R ; G, \lambda)\right)=0$. Then, passing to a refinement of $\left\{C_{i}\right\}_{i \in l}$ if need be, we have $v \sim 0$. That is, there are functions $\mu_{i j}: C_{i j} \rightarrow \mathbb{T}$ such that

$$
v_{i j k}([u])=\mu_{i j}([u]) \mu_{j k}([u]) \overline{\mu_{i k}([u])} .
$$

Then replacing $\phi_{i j}$ by $\mu_{i j} \cdot \phi_{i j}$, we can assume that $v_{i j k} \equiv 1$. In particular, (5.6) implies that

$$
h_{j k} \circ h_{i j}=h_{i k} \quad \text { for all triples } i, j, k \in I \text {. }
$$

Then we define

$$
\Lambda=\coprod_{i \in} G_{F_{i}} / \sim
$$

where $(i, \gamma) \sim\left(j, h_{i j}(\gamma)\right)$ if $r(\gamma) \in\left[F_{i j}\right]$. (Note that if $r(\gamma) \in\left[F_{i j}\right]$ and $s(\gamma) \in F_{i}$, then actually $s(\gamma) \in F_{i j}$ as $s(\gamma) \sim r(\gamma) \sim u$ with $u \in p^{-1}\left(C_{i j}\right)$.)

Observe that $\Lambda$ is a $\mathbb{T}$-space: $t \cdot[i, \gamma]=[i, t \cdot \gamma]$. (This is well defined because

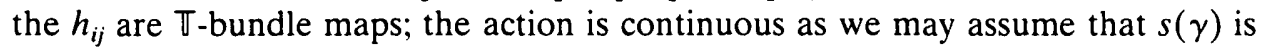
in the interior of $F_{i}$, and hence that there is a neighbourhood of $\gamma$ in $G$ which is contained in $\left.G_{F_{i}}\right)$ Define $\pi: \Lambda \rightarrow X$ by $\pi([i, \gamma])=r(\gamma)$. (This map is clearly well defined and it is continuous and open for the same reasons as described above.) With this structure $\Lambda$ is a $\mathbb{T}$-bundle over $X$.

Recall that $\varepsilon(\Lambda)=G_{\pi}=\left.\widetilde{G}_{\pi}\right|_{R}$, where $\widetilde{G}_{\pi}$ is $\Lambda * \Lambda / \mathbb{T}$. Alternatively,

$$
G_{\pi}=\left(\left.\Lambda * \Lambda\right|_{R}\right) / \mathbb{T}
$$

It will be convenient to define maps $\tilde{h}_{i j}: G^{F_{i j}} \rightarrow G^{F_{i j}}$ by the formula

$$
\begin{aligned}
\tilde{h}_{i j}(\gamma) & =h_{i j}\left(\gamma^{-1}\right)^{-1}=\left(\gamma^{-1} \phi_{i j}(r(\gamma))\right)^{-1} \\
& =\phi_{i j}(r(\gamma))^{-1} \gamma=\phi_{i j}(r(\gamma)) \gamma .
\end{aligned}
$$

Note that we still have $\tilde{h}_{j k} \circ \tilde{h}_{i j}=\tilde{h}_{i k}$. We define $\Gamma$ to be the quotient of the disjoint union $\amalg_{i} G_{F_{i}} * G^{F_{i}}$ where $[i, \gamma, \beta] \sim\left[j, h_{i j}(\gamma), \tilde{h}_{i j}(\beta)\right]$. The point is that we claim that $\Gamma$ is homeomorphic to $\left.\Lambda * \Lambda\right|_{R}$. To see, this, suppose $\left.([i, \gamma],[j, \beta]) \in \Lambda * \Lambda\right|_{R}$. Then $r(\gamma) \sim r(\beta)$ and $s(\beta) \in F_{j i}$. Therefore $h_{i j}(\beta)$ is defined. Thus if $[i, \gamma]=$ $\left[k, \gamma^{\prime}\right]$ and $[j, \beta]=\left[l, \beta^{\prime}\right]$, then $\gamma, \beta \in\left[F_{i j k l}\right]$ and $\gamma^{\prime}=h_{i k}(\gamma)$ and $\beta^{\prime}=h_{j l}(\beta)$. But

$$
\tilde{h}_{l k}\left(h_{j l}(\beta)^{-1}\right)=\tilde{h}_{l k}\left(\tilde{h}_{j l}\left(\beta^{-1}\right)\right)=\tilde{h}_{j k}\left(\beta^{-1}\right)=\tilde{h}_{i k}\left(\tilde{h}_{j i}\left(\beta^{-1}\right)\right) .
$$

Thus there is a well-defined map $\psi$ from $\left.\Lambda * \Lambda\right|_{R}$ to $\Gamma$ given by $\psi([i, \gamma],[j, \beta])=$ $\left[i, \gamma, \tilde{h}_{j i}\left(\beta^{-1}\right)\right]$. (Since $r(\gamma) \sim r(\beta)$ and $r\left(\tilde{h}_{j i}\left(\beta^{-1}\right)\right)=r\left(\phi_{j i}(s(\beta))\right)=s(\beta)$, we have $s(\gamma)=r\left(\tilde{h}_{j i}\left(\beta^{-1}\right)\right)$, so $\left(\gamma, \tilde{h}_{j i}\left(\beta^{-1}\right)\right) \in G_{F_{i}} * G^{F_{i}}$.) Since the interiors of the $C_{i}$ cover $X / R, \psi$ is clearly continuous, open, and surjective. To see that it is injective as well, suppose that $\psi([i, \gamma],[j, \beta])=\psi\left(\left[k, \gamma_{1}\right],\left[l, \beta_{1}\right]\right)$. Then $\left[i, \gamma, \tilde{h}_{j i}\left(\beta^{-1}\right)\right]=$ $\left[k, \gamma_{1}, \tilde{h}_{l k}\left(\beta_{1}^{-1}\right)\right]$. Therefore $\gamma_{1}=h_{i k}(\gamma)$ and $\tilde{h}_{l k}\left(\beta_{1}^{-1}\right)=\tilde{h}_{i k}\left(\tilde{h}_{j i}\left(\beta^{-1}\right)\right)=\tilde{h}_{j k}\left(\beta^{-1}\right)$; thus $\beta_{1}^{-1}=\tilde{h}_{k l}\left(\tilde{h}_{j k}\left(\beta^{-1}\right)\right)=\tilde{h}_{j l}\left(\beta^{-1}\right)$. In other words, $[i, \gamma]=\left[k, \gamma_{1}\right]$ and $[j, \beta]=$ $\left[l, \beta_{1}\right]$. This proves the claim.

Note that $\Gamma$ is a $T$-space in a natural way: $t \cdot[i, \gamma, \beta]=[i, t \cdot \gamma, \bar{t} \cdot \beta]$, and that $\psi$ is equivariant with respect to this action: $\psi\left(t \cdot\left[[i, \gamma],[j, \beta]^{*}\right]\right)=$ $\psi\left([i, t \cdot \gamma],[j, t \cdot \beta]^{*}\right)=\left[i, t \cdot \gamma, \bar{t} \cdot \tilde{h}_{j i}\left(\beta^{-1}\right)\right]=t \cdot \psi\left([i, \gamma],[j, \beta]^{*}\right)$. Thus we can 
identify $\varepsilon(\Lambda)$ with $\Gamma / \mathbb{T}$. Furthermore, if $(\gamma, \beta) \in G_{F_{i}} * G^{F_{i}}$, then $s(\gamma)=u=r(\beta)$. Therefore

$$
\begin{aligned}
h_{i j}(\gamma) \tilde{h}_{i j}(\beta) & =h_{i j}(\gamma) h_{i j}\left(\beta^{-1}\right)^{-1} \\
& =\gamma \phi_{i j}(u)\left(\beta^{-1} \phi_{i j}(u)\right)^{-1}=\gamma \beta .
\end{aligned}
$$

Thus the formula $[i, \gamma, \beta] \mapsto \gamma \beta$ defines a map $\Theta$ from $\Gamma$ to $G$. Again since the interiors of the $C_{i}$ cover $X / R$ and since $G$ is a T-groupoid, we can apply Theorem 2.5 to conclude that $\Theta$ is an open surjection. Then it is straightforward to check that $\Theta$ factors through $\Gamma / \mathbb{T}$ and so defines a homeomorphism of $\varepsilon(\Lambda)$ with $G$. We have shown that $\operatorname{ker} \delta \subseteq \operatorname{Im} \varepsilon$.

Now suppose that $q: \Lambda \rightarrow X$ is a circle bundle. Let $H=X / R \times \mathbb{T}$ be the trivial group bundle groupoid. We view $\Lambda$ as a right $H$-space: let $\Lambda * H=$ $\{(\lambda,([x], t)):[q(\lambda)]=[x]\}$, and define $\lambda \cdot([x], t)=t \cdot \lambda$. Then $\Lambda$ is a free and proper $H$-space and we let $G$ be the imprimitivity groupoid as described in $\S 3$. (Here $\sigma=p \circ q$.) That is, $G=\Lambda * \Lambda / H$, where

$$
\begin{gathered}
\Lambda * \Lambda=\left\{\left(\lambda_{1}, \lambda_{2}\right):\left[q\left(\lambda_{1}\right)\right]=\left[q\left(\lambda_{2}\right)\right]\right\}, \\
(\Lambda * \Lambda) * H=\left\{\left(\left(\lambda_{1}, \lambda_{2}\right),([x], t)\right):\left[q\left(\lambda_{1}\right)\right]=\left[q\left(\lambda_{2}\right)\right]=[x]\right\},
\end{gathered}
$$

and

$$
\left(\lambda_{1}, \lambda_{2}\right) \cdot([x], t)=\left(t \cdot \lambda_{1}, t \cdot \lambda_{2}\right) .
$$

Then $H$ and $G$ are equivalent groupoids (Theorem 3.5) and $\delta\left(C^{*}(G, \lambda)\right)=0$ by [9, Theorem 2.8]. However, the identity map on $\Lambda \times \Lambda$ induces a topological groupoid isomorphism between $G$ and $\varepsilon(\Lambda)$, and since $C^{*}(R ; \varepsilon(\Lambda), \lambda)$ is a quotient of $\mathrm{C}^{*}(G, \lambda)$, we have $\delta\left(\mathrm{C}^{*}(R ; \varepsilon(\Lambda), \lambda)\right)=0$ as well. This completes the proof of exactness at $\mathrm{Tw}(R)$.

\section{Exactness at $H^{2}(X / R, \mathscr{S})$}

To show that $\operatorname{Im}(\delta) \subseteq \operatorname{ker}\left(p^{*}\right)$, we need to consider $[\tilde{v}]=p^{*}([v])$, where $v=\left\{v_{i j k}\right\}$ is a cocycle determined by the local Morita equivalences of $\mathrm{C}^{*}(R ; G, \lambda)$ for some $\mathbb{T}$-groupoid over $R$ as in equation (5.6) corresponding to sections $\omega_{i}$, a cover $\left\{C_{i}\right\}_{i \in l}$, and data $F_{i}, \phi_{i j}$, etc., as before. Then $\widetilde{v}=\left\{\tilde{v}_{i j k}\right\}$ is defined on the cover $\left\{D_{i}\right\}_{i \in I}=\left\{p^{-1}\left(C_{i}\right)\right\}_{i \in I}$ by the formula $\bar{v}_{i j k}(x)=v_{i j k}(p(x))$. Notice that we can take refinements $\left\{D_{a}\right\}_{a \in A}$ of $\left\{D_{i}\right\}$ at will-with each $D_{a}$ not necessarily saturatedand we still have $\widetilde{v}_{a b c}$ determined by

$$
\left(\omega_{a}(p(x)), \widetilde{v}_{a b c}(x)\right)=\phi_{a b}\left(\omega_{a}(p(x))\right) \phi_{b c}\left(\omega_{b}(p(x))\right) \phi_{a c}\left(\omega_{a}(p(x))\right)^{-1} .
$$

(We are abusing notation here. Formally, one must choose a refining map $\zeta: A \rightarrow I$ so that $D_{a} \subset D_{\zeta(a)}$, and then $\widetilde{v}_{a b c}$ is the restriction of $\tilde{v}_{\zeta(a) \zeta(b) \zeta(c)}$ to $D_{a b c}$. There are (possibly) many such maps $\zeta$, but it is a standard part of the theory that the cohomology class of $\tilde{v}$ is independent of the choice of refining map.)

Next observe that as $\left.G\right|_{\left[F_{i}\right]}$ is a $\mathbb{T}$-groupoid, and applying Theorem 2.5, it follows that $\left.r\right|_{G_{F_{i}}}: G_{F_{i}} \rightarrow\left[F_{i}\right]$ is open. Thus $\left.r\right|_{G_{F_{i}}}$ is a $\mathbb{T}$-bundle and must be locally trivial [5]. Since the interiors of the $\left[F_{i}\right] \operatorname{cover} X$, each $u \in X$ has a neighbourhood $D$ contained in some $\left[F_{i}\right]$ over which $\left.r\right|_{G_{F_{i}}}$ is the trivial bundle. Then there is a map $\alpha: D \rightarrow G_{F_{i}}$ such that $r(\alpha(y))=y$ and $s(\alpha(y))=\omega_{i}(p(y))$. (Recall that $F_{i}$ is a transversal for $\left[F_{i}\right]$ and $s(\alpha(y)) \in F_{i}$ and is equivalent to $r(\alpha(y))=y$.) 
It follows that there are a refinement $\left\{D_{a}\right\}_{a \in A}$ of $\left\{D_{i}\right\}_{i \in I}$ and continuous maps $\alpha_{a}: D_{a} \rightarrow G$ such that $r\left(\alpha_{a}(u)\right)=u$ and $s\left(\alpha_{a}(u)\right)=\omega_{a}(p(u))$. If we define $\beta_{a b}=$ $\alpha_{a}(x)^{-1} \alpha_{b}(x)$, then

and

$$
r\left(\beta_{a b}(x)\right)=\omega_{a}(p(x))=r\left(\phi_{a b}\left(\omega_{a}(p(x))\right)\right),
$$

$$
s\left(\beta_{a b}(x)\right)=\omega_{b}(p(x))=s\left(\phi_{a b}\left(\omega_{a}(p(x))\right)\right) .
$$

Therefore there are continuous functions $\mu_{a b}: D_{a b} \rightarrow \mathbb{T}$ such that

$$
\phi_{a b}\left(\omega_{a}(p(x))\right)=\mu_{a b}(x) \beta_{a b}(x) .
$$

Plugging (6.2) into (6.1) yields

$$
\tilde{v}_{a b c}(x)=\mu_{a b}(x) \mu_{b c}(x) \overline{\mu_{a c}(x)} .
$$

(Here we have used the fact that $\beta_{a b}(x) \beta_{b c}(x) \beta_{a c}(x)^{-1}=\omega_{a}(p(x))$, a unit.) Thus, $\widetilde{v}$ cobounds and $p^{*}([v])=0$ as desired.

To complete the proof of Theorem 1.1 we now only need to show that if $\delta \in H^{2}(X / R, \mathscr{S})$ satisfies $p^{*}(\delta)=0$, then there is a T-groupoid $G$ over $R$ such that $\delta\left(C^{*}(R ; G, \lambda)\right)=\delta$.

Fix a locally finite open cover $\mathfrak{H}=\left\{U_{a}\right\}_{a \in A}$ of $X / R$ and an alternating (see [16, Lemma 3.4]) cocycle $v=\left\{v_{i j k}\right\} \in Z^{2}(\mathfrak{A}, \mathscr{S})$ which satisfies $[v]=\delta$. By assumption, the cocycle $\widetilde{v}$ in $Z^{2}\left(p^{-1}(\mathscr{Q}), \mathscr{S}\right)$ defined by $\widetilde{v}_{a b c}(x)=v_{a b c}(p(x))$ defines the trivial class in $H^{2}(X, \mathscr{S})$. Therefore there is a locally finite refinement $\mathfrak{B}=\left\{V_{i}\right\}_{i \in l}$ of $p^{-1}(\mathscr{A})$ such that (an image of) $\tilde{v}$ is actually trivial in $Z^{2}(\mathfrak{B}, \mathscr{S})$. To be precise, we assume that there is a refining map $\zeta: I \rightarrow A$, which we fix, so that $V_{i} \subseteq p^{-1}\left(U_{\zeta(i)}\right)$ for all $i \in I$, and so that there are continuous functions $\mu_{i j}: V_{i j} \rightarrow \mathbb{T}$ such that

$$
\mu_{i j}(x) \mu_{j k}(x) \overline{\mu_{i k}(x)}=v_{\zeta(i) \zeta(j) \zeta(k)}(p(x))
$$

for all $x \in V_{i j k}$.

Next we form the groupoid version of the Raeburn-Taylor algebra with Dixmier-Douady invariant $\delta$. To do this, let $Z=\bigsqcup_{a \in A} U_{a}$, and define $\psi: Z \rightarrow$ $X / R$ by $\psi(a, u)=u$. Let $R(\psi)$ be the induced relation in $Z \times Z$. The RaeburnTaylor groupoid $\Gamma$ is the extension of $R(\psi)$ determined by the continuous 2-cocycle $\sigma \in H^{2}(R(\psi), \mathbb{T})$ defined by

$$
\sigma(((a, u),(b, u)),((b, u),(c, u)))=\tilde{v}_{a b c}(u) .
$$

Thus $\Gamma=R(\psi) \times \mathbb{T}$ with multiplication defined by

$$
((a, u),(b, u), s) \cdot((b, u),(c, u), t)=\left((a, u),(c, u), s t \tilde{v}_{a b c}(u)\right) .
$$

The point is that $\Gamma$ is a $\mathbb{T}$-groupoid (even a twist in Kumjian's sense) over $R(\psi)$ and $\delta=\delta\left(C^{*}(R(\psi) ; \Gamma, c)\right)$, where $c$ is the Haar system given by the usual collection counting measures (see $[\mathbf{1 4}, \mathbf{6}]$ ).

We now define a family of maps which Kumjian calls a $\Gamma$-cocycle on $X$. We define $\theta_{i j}: V_{i j} \rightarrow \Gamma$ by

$$
\theta_{i j}(x)=\left((\zeta(i), p(x)),(\zeta(j), p(x)), \overline{\mu_{i j}(x)}\right) .
$$

The crucial properties that the $\theta_{i j}$ enjoy are as follows:

(1) $\left(\theta_{i j}(x), \theta_{j k}(x)\right) \in \Gamma^{(2)}$ for all $x \in V_{i j k}$, and 
(2) $\theta_{i j}(x) \theta_{j k}(x)=\theta_{i k}(x)$.

Property (1) is clear by construction, while Property (2) follows from (6.3) and (6.4).

Now set $W=\bigsqcup_{i \in I} V_{i}$, and put $Y_{0}=\Gamma * W=\left\{(\gamma, i, x): s(\gamma)=\theta_{i i}(x)\right\}$. To see that $Y_{0}$ is non-empty, notice that $\mu_{i i}$ is identically 1 since $v$ is alternating (consider the equation $\left.v_{\zeta(i) \zeta(i) \zeta(j)}(p(x))=1\right)$, and therefore

$$
\theta_{i i}(x)=\left((\zeta(i), p(x)),(\zeta(i), p(x)), \mu_{i i}(x)\right) \in \Gamma^{(0)} .
$$

Let $R(\theta)$ be the collection of triples $(i, x, j)$ with $x \in V_{i j}$. Then $R(\theta)$ has a natural groupoid structure coming from viewing $R(\theta)$ as the equivalence relation in $W \times W$ induced by $\phi: W \rightarrow X$ where $\phi(i, x)=x$. We claim that $Y_{0}$ is a free and proper $R(\theta)$-space. To see this, define $\tilde{\sigma}: Y_{0} \rightarrow W\left(=R(\theta)^{(0)}\right)$ by $\widetilde{\sigma}(\gamma, i, x)=(i, x)$. To show that $\tilde{\sigma}$ is open it suffices to show that any net $\left\{\left(i, x_{\alpha}\right): x_{\alpha} \in V_{i}\right\}$ converging to $(i, x)$ with $x \in V_{i}$ has a subnet which lifts to $Y_{0}$. (Here, the fact that $W$ is a disjoint union of open sets allows us to consider a fixed $i \in I$.) But $\theta_{i i}\left(x_{\alpha}\right)$ converges to $\theta_{i i}(x)$ in $\Gamma^{(0)}$, and $s: \Gamma \rightarrow \Gamma^{(0)}$ is open. Thus, passing to a subnet and relabelling, we may find $\gamma_{\alpha} \in \Gamma$ with $s\left(\gamma_{\alpha}\right)=\theta_{i i}\left(x_{\alpha}\right)$. Thus, $\left(\gamma_{\alpha}, i, x_{\alpha}\right) \in Y_{0}$. We define

$$
(\gamma, i, x) \cdot(i, x, j)=\left(\gamma \theta_{i j}(x), x, j\right) .
$$

(Notice that $\left(\gamma, \theta_{i j}(x)\right) \in \Gamma^{(2)}$ as $s(\gamma)=\theta_{i i}(x)$, and $\left(\theta_{i i}(x), \theta_{i j}(x)\right) \in \Gamma^{(2)}$ by construction.) The fact that this action is free and proper is straightforward to verify.

Therefore $Y(\theta)=Y_{0} / R(\theta)$ is a locally compact Hausdorff space. We will write $[\gamma, i, x]$ for the class of $(\gamma, i, x)$ in $Y(\theta)$. The map $\sigma: Y(\theta) \rightarrow X$ given by $\sigma([\gamma, i, x])=x$ is clearly well defined, continuous, and open. Similarly, $\rho: Y(\theta) \rightarrow$ $X / R$ given by $\rho([\gamma, i, x])=r(\gamma)$ is well defined and continuous. To see that $\rho$ is open, it will suffice to show that any convergent net $r\left(\gamma_{\alpha}\right) \rightarrow r(\gamma)$ has a subnet which lifts to $Y(\theta)$. Since $r$ is open, we may assume that $\gamma_{\alpha} \rightarrow \gamma$. Thus $s\left(\gamma_{\alpha}\right) \rightarrow s(\gamma)$. Let $s(\gamma)=(\zeta(i), u)$, and we may assume that $s\left(\gamma_{\alpha}\right)=\left(\zeta(i), u_{\alpha}\right)$. Since $p$ is open, we can assume that there are $x_{\alpha} \in V_{i}$ converging to $x \in V_{i}$ with $p\left(x_{\alpha}\right)=u_{\alpha}$. Thus, $\left\{\left(\gamma_{\alpha}, i, x_{\alpha}\right)\right\}$ is the required net.

We make $Y(\theta)$ into a left- $\Gamma$-space, with respect to $\rho$, in the obvious way: if $s(\alpha)=r(\gamma)=\rho([\gamma, i, x])$, then $\alpha \cdot[\gamma, i, x]=[\alpha \gamma, i, x]$. Using the fact that the natural map $(\gamma, i, x) \mapsto[\gamma, i, x]$ is open, it is not difficult to show that the action is continuous. It is clearly free, and properness is a consequence of the following observation. Suppose that $\left[\gamma_{\lambda}, i_{\lambda}, x_{\lambda}\right] \rightarrow[\gamma, i, x]$ and $\left[\alpha_{\lambda} \gamma_{\lambda}, i_{\lambda}, x_{\lambda}\right] \rightarrow\left[\gamma^{\prime}, i, x\right]$. Since the natural map is open, we can pass to a subnet and relabel and assume that $i_{\lambda}$ is eventually equal to $i$ and that there are $\gamma_{\lambda}^{\prime}$ with $\left[\gamma_{\lambda}^{\prime}, i, x_{\lambda}\right]=\left[\gamma_{\lambda}, i, x_{\lambda}\right]$ with the property that $\left(\gamma_{\lambda}^{\prime}, i, x_{\lambda}\right) \rightarrow(\gamma, i, x)$ as well as $\left(\alpha_{\lambda} \gamma_{\lambda}^{\prime}, i, x_{\lambda}\right) \rightarrow\left(\gamma^{\prime}, i, x\right)$. (Recall that $\theta_{i i}\left(x_{\lambda}\right)$ is a unit.) Thus $\alpha_{\lambda}$ must converge to $\gamma^{\prime} \gamma^{-1}$ as desired.

\section{Lemma 6.1. The map $\sigma$ induces a homeomorphism of $\Gamma \backslash Y(\theta)$ onto $X$.}

Proof. Let $\overline{[\gamma, i, x]}$ denote the $\Gamma$-orbit of $[\gamma, i, x]$. Since $\sigma$ is constant on $\overline{[\gamma, i, x]}$, the map $\overline{[\gamma, i, x]} \mapsto x$ is well defined. Continuity and openness follow from the continuity and openness of $\sigma$ and the natural map of $Y(\theta)$ onto $\Gamma \backslash Y(\theta)$. If $x \in X$, say $x \in V_{i}$, then there is a $\gamma \in \Gamma$ with $s(\gamma)=\theta_{i i}(x)$. Then $(\gamma, i, x) \in Y_{0}$ and $\overline{[\gamma, i, x]} \mapsto x$. This proves surjectivity. Finally, suppose that $[\gamma, i, x]$ and $\left[\gamma^{\prime}, j, x\right]$ 
are in $Y(\theta)$. Then $x \in V_{i j}$ and $[\gamma, i, x]=\left[\gamma \theta_{i j}(x), j, x\right]$. But $s\left(\gamma^{\prime}\right)=\theta_{j j}(x)=$ $s\left(\gamma \theta_{i j}(x)\right)$. Thus $\gamma^{\prime}\left(\gamma \theta_{i j}(x)\right)^{-1} \in \Gamma$. Thus $\overline{[\gamma, i, x]}=\overline{\left[\gamma^{\prime}, j, x\right]}$, and this proves injectivity.

Now we let $G$ be the imprimitivity groupoid for the left action of $\Gamma$ on $Y(\theta)$ (see Theorem 3.5 and Remark 3.6). Recall that $G$ is the quotient of $Y(\theta) * Y(\theta)=\left\{\left(y_{1}, y_{2}\right): \rho\left(y_{1}\right)=\rho\left(y_{2}\right)\right\}$ by the diagonal $\Gamma$-action. Using Lemma 6.1, we identify $G^{(0)}=\Gamma \backslash Y(\theta)$ with $X$ via the map sending $x \in V_{i} \subseteq X$ to $\left[\left[\theta_{i i}(x), i, x\right],\left[\theta_{i i}(x), i, x\right]\right]$. Then $s_{G}\left(\left[y_{1}, y_{2}\right)\right]=\sigma\left(y_{2}\right)$ and $r_{G}\left(\left[y_{1}, y_{2}\right]\right)=\sigma\left(y_{1}\right)$.

It now follows from Theorem 3.5 that $G$ is a $\mathbb{T}$-groupoid over an equivalence relation $R_{G}=j_{G}(G)$ in $X \times X$. Observe that if $\left[(\gamma, i, x),\left(\gamma^{\prime}, j, x^{\prime}\right)\right] \in G$, then $r(\gamma)=r\left(\gamma^{\prime}\right)$ while $s(\gamma)=\theta_{i i}(x)$ and $s\left(\gamma^{\prime}\right)=\theta_{j j}\left(x^{\prime}\right)$. This implies that $\left(s(\gamma), s\left(\gamma^{\prime}\right)\right)$ belongs to $R(\psi)$, or more simply, $p(x)=\psi(s(\gamma))=\psi\left(s\left(\gamma^{\prime}\right)\right)=p\left(x^{\prime}\right)$. In sum, if $\left[y_{1}, y_{2}\right] \in G$, then $j_{G}\left(\left[y_{1}, y_{2}\right]\right)=\left(r_{G}\left(\left[y_{1}, y_{2}\right]\right), s_{G}\left(\left[y_{1}, y_{2}\right]\right)\right)$ belongs to $R$. To show that $j$ is surjective, suppose that $p(x)=p(y)$ (so that $(x, y) \in R$ ). Then $x \in V_{i}$ and $y \in V_{j}$ for appropriate $i, j \in I$. Then $p(x) \in U_{\zeta(i) \zeta(j)}$ and $((\zeta(i), p(x)),(\zeta(j), p(y))) \in R(\psi)$. Let $\quad \gamma_{1}=((\zeta(i), p(x)),(\zeta(i), p(x)), 1)$ and $\gamma_{2}=((\zeta(i), p(x)),(\zeta(j), p(y)), 1)$. Then $\left(\left[\gamma_{1}, i, x\right],\left[\gamma_{2}, j, y\right]\right) \in Y(\theta) * Y(\theta)$ and surjectivity follows.

We have shown that $[G] \in \operatorname{Tw}(R)$, and that $G$ is equivalent to $\Gamma$ in such a way

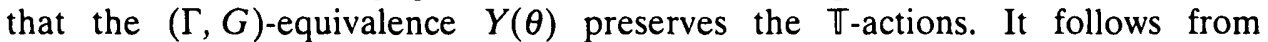
Theorem 3.2 that $\mathrm{C}^{*}(R(\psi) ; \Gamma, c)$ is strongly Morita equivalent to $\mathrm{C}^{*}(R ; G, \lambda)$. This implies that $\delta([G])=\delta$, which completes the proof of exactness at $H^{2}(X / R, \mathscr{S})$, and completes the proof of Theorem 1.1 .

\section{References}

1. C. Albert and P. Dazord, 'Groupoïdes de Lie and groupoïdes symplectiques', Symplectic geometry, groupoids, and integrable systems (eds P. Dazord and A. Weinstein), MSRI Publications 20 (Springer, New York, 1991), pp. 1-12.

2. A. Coste, P. Dazord, and A. Weinstein, 'Groupoïdes symplectiques', Pub. Dép. Math. (Lyon) (1987) $1-62$.

3. J. DiXmiER, $C^{*}$-algebras (North-Holland, New York, 1977).

4. J. DiXmier and A. DouAdy, 'Champs continus d'espaces hilbertiens et de C*-algèbres', Bull. Soc. Math. France 91 (1963) 227-284.

5. A. Gleason, 'Spaces with a compact Lie group of transformations', Proc. Amer. Math. Soc. 1 (1950) 35-43.

6. A. KumJIAN, 'Diagonals in algebras of continuous trace', Operator algebras and their connections with topology and ergodic theory, Conference proceedings, Buşteni, Romania (1983), Lecture 7. A. Kumulan, 'On C*-diagonals', Canad. J. Math. 38 (1986) 969-1008.

8. K. MACKENZIE, Lie groupoids and Lie algebroids in differential geometry, London Mathematical Society Lecture Note Series 124 (Cambridge University Press, 1987).

9. P. S. Muhly, J. Renault, and D. P. Williams, 'Equivalence and isomorphism for groupoid C*-algebras', J. Operator Theory 17 (1987) 3-22.

10. P. S. MUHLY and D. P. WiLliams, 'Transformation group C*-algebras with continuous trace. Il', J. Operator Theory 11 (1984) 109-124.

11. P. S. Muhly and D. P. Williams, 'Continuous trace groupoid C*-algebras', Math. Scand. 66 (1990) 231-241.

12. P. S. Muhly and D. P. Williams, 'Continuous trace groupoid C*-algebras. II', Math. Scand. 70 (1992) 127-145.

13. D. Olesen and I. Raeburn, 'Pointwise unitary automorphism groups', J. Funct. Anal. 93 (1990) 278-309.

14. I. RAEBURN and J. L. TAYLOR, 'Continuous-trace $C^{*}$-algebras with given Dixmier-Douady class', J. Austral Math. Soc. Ser. A 38 (1985) 394-407. 
15. 1. RaEbURN and D. P. Williams, 'Pull-backs of $\mathrm{C}^{*}$-algebras and crossed products by certain diagonal actions', Trans. Amer. Math. Soc. 287 (1985) 755-777.

16. I. RaEBURN and D. P. Williams, 'Topological invariants associated to the spectrum of crossed product C*-algebras', J. Funct. Anal. 116 (1993) 245-276.

17. I. Raeburn and D. P. Williams, 'Dixmier-Douady classes for dynamical systems and crossed products', Canad. J. Math. 45 (1993) 1032-1066.

18. J. ReNAULT, $A$ groupoid approach to $C^{*}$-algebras, Lecture Notes in Mathematics 793 (Springer, Berlin, 1980).

19. J. RENAULT, 'Two applications of the dual groupoid of a $\mathrm{C}^{*}$-algebra', Operator algebras and their connections with lopology and ergodic theory, Conference proceedings, Buşteni, Romania (1983), Lecture Notes in Mathematics 1132 (Springer, Berlin, 1985), pp. $434-445$. 022..(1983), Lecture Notes in Mathematics 1132 (Springer, Berlin, 1985), pp. 434-445.

20. J. RENAULT, 'Représentations des produits croisés d'algèbres de groupoïdes', $J$. Operator Theory 18 (1987) 67-97.

21. M. A. RiEfFEL, 'Induced representations of C*-algebras', Adv. in Math. 13 (1974) 176-257.

22. M. A. RIEFFEL, 'Application of strong Morita equivalence to transformation group C*-algebras', Proceedings of Symposia in Pure Mathematics 38, Part I (American Mathematical Society, Providence, R.I., 1982), pp. 299-310.

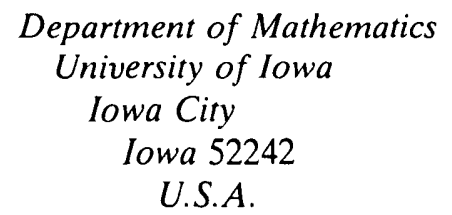

\author{
Department of Mathematics \\ Dartmouth College \\ Hanover \\ New Hampshire 03755 \\ U.S.A.
}

